\title{
Clean Coal III Project: Blast Furnace Granular Coal Injection Project \\ Trial 1 Report - Blast Furnace Granular Coal Injection - Results with Low Volatile Coal
}

\author{
Topical Report \\ November 1, 1998
}

Work Performed Under Contract No.: DE-FC21-91MC27362

For

U.S. Department of Energy

Office of Fossil Energy

Federal Energy Technology Center

P.O. Box 880

Morgantown, West Virginia 26507-0880

By

Bethlehem Steel Corporation

Bethlehem, Pennsylvania 18016 


\section{Disclaimer}

This report was prepared as an account of work sponsored by an agency of the United States Government. Neither the United States Government nor any agency thereof, nor any of their employees, makes any warranty, express or implied, or assumes any legal liability or responsibility for the accuracy, completeness, or usefulness of any information, apparatus, product, or process disclosed, or represents that its use would not infringe privately owed rights. Reference herein to any specific commercial product, process, or service by trade name, trademark, manufacturer, or otherwise does not necessarily constitute or imply its endorsement, recommendation, or favoring by the United States Government or any agency thereof. The views and opinions of authors expressed herein do not necessarily state or reflect those of the United States Government or any agency thereof. 


\section{DISCLAIMER}

Portions of this document may be illegible in electronic image products. Images are produced from the best available original document. 


\section{TABLE OF CONTENTS}

\section{Page}

INTRODUCTION

BACKGROUND 1

BLAST FURNACE OPERATIONS

FURNACE OPERATING CONDITIONS

ENVIRONMENTAL TEST RESULTS

FURNACE THERMAL CONDITIONS AND 5

LINING WEAR

DISCUSSION 


\section{LIST OF TABLES}

Table 1 Base Period Evaluation - Burns Harbor C Furnace - Summary of Operations

Table 2 Burns Harbor C Furnace Injected Coal Analysis and Sizing

Table 3 Burns Harbor C Furnace Sulfur Balance

\section{LIST OF FIGURES}

Figure 1 Burns Harbor C Furnace - Permeability \& Injected Coal Rate

Figure 2 Blast Furnace Recirculating Water System

Figure 3 Burns Harbor C Furnace - Inwall Refractory Temperatures

Figure 4 Burns Harbor C Furnace Thermal Loads

Figure 5 Lining Wear vs. Coal Injection

Figure 6 Comparisons of Lining Wear

Figure 7 Burns Harbor C \& D Blast Furnaces

\section{APPENDICES}

Appendix I Gaseous Stream Testing Results

Appendix II Wastewater Monitoring Summaries 


\section{BLAST FURNACE GRANULAR COAL INJECTION - RESULTS WITH LOW VOLATILE COAL}

\section{INTRODUCTION}

This report describes the first coal trial test conducted with the Blast Furnace Granular Coal Injection System at Bethlehem Steel Corporation's Burns Harbor Plant. This demonstration project is divided into three phases:

$$
\begin{aligned}
& \text { Phase I - Design } \\
& \text { Phase II - Construction } \\
& \text { Phase III - Operation }
\end{aligned}
$$

The design phase was conducted in 1991-1993. Construction of the facility began in August 1993 and was completed in late 1994. The coal injection facility began operating in January 1995 and Phase III began in November 1995.

The Trial 1 base test on C furnace was carried out in October 1996 as a comparison period for the analysis of the operation during subsequent coal trials.

\section{BACKGROUND}

The granulated coal injection facility at the Burns Harbor Plant began operation in January 1995. Coal injection began on D furnace in mid-December 1994, primarily to test the coal grinding and preparation circuits. Significant operations began January 19, 1995 when coal was injected through four tuyeres at a total rate of 20 pounds/NTHM. Coal injection was initiated on C furnace on February 9, 1995 using four tuyeres at an overall rate of 25 pounds/NTHM. The remaining 24 tuyeres used natural gas injection at the same time. These conditions were maintained throughout February and March. Operating difficulties with the coal grinding and preparation system, typical of new facility start up problems, required equipment changes and modifications. The first complete month of operation with coal as the sole injectant on C furnace was June 1995. On D furnace, complete coal injection began in April 1995. Since that time an operational learning curve and the development of efficient operating practices with the granulated coal facility were completed.

Sydney coal, a high volatile coal, was used on both furnaces for eight months. Six different low volatile coal types were subsequently used on both furnaces for seven months. The good operational experience with the low volatile coal resulted in a decision to use low volatile Virginia Pocahontas coal as the standard for granulated coal injection at Burns Harbor. 
The objective of the overall test program is to determine the effect of coal grind and coal type on blast furnace performance. Meaningful analysis of blast furnace process changes that occur with a change of injected coal type or sizing requires a base test period from which comparisons can be made for future tests. The requirements for an acceptable trial are:

1. The base period used for comparison should be chronologically close to the ensuing trial period.

2. A steady state operation with minimum day-to-day variability. The length of the test period is flexible, however, the longer the trial duration, the more definitive the results.

3. A minimum of major furnace process changes during the trial, particularly with the process variable that is being evaluated.

\section{BLAST FURNACE OPERATIONS}

The Burns Harbor $\mathrm{C}$ furnace operation during October 1996 meets the requirements for an acceptable comparative base period. The operating results for this period may be used as the basis for the evaluation of future trials.

The October operation on $\mathrm{C}$ furnace was adequate in terms of furnace performance parameters using coal injection. The injection facility supplied coal without interruption for the entire month. The average rate of 264 pounds/NTHM varied from 246-278 pounds/NTHM on a daily basis. The furnace coke rate during the period averaged 661 pounds/NTHM.

The important furnace operating conditions that indicate the full range of furnace performance results are discussed and documented in the following. In addition, extensive environmental stream testing of the closed water and gas cleaning systems, furnace refractory temperatures, thermal loads and refractory wear are presented for the Trial Base period.

\section{FURNACE OPERATING CONDITIONS}

The $C$ furnace is designated as the granulated coal test facility due, in large part, to the physical improvements made to the furnace during the 1994 reline. The $\mathrm{C}$ furnace was enlarged slightly and the refractory cooling system was upgraded to a high density plate cooling configuration. The furnace stack region on $\mathrm{C}$ has closely spaced cooling plates that are not on the $\mathrm{D}$ furnace. This high density cooling was specifically designed for the rigors of high coal injection rates and to provide for increased production capability. 
The essential operating characteristics for the base test are shown in Table 1 . These values comprise the operating comparative base results necessary for future trial evaluation.

The type of coal used and the grind size distribution for the trial is of primary consideration for this period. The monthly average chemistry for the Virginia Pocahontas injected coal is shown on Table 2. This coal is a low volatile type with high carbon and relatively low ash content. These two characteristics should provide the highest coke replacement value for the furnace process. The gross heating value, GHV, is also an indication of the heat value provided in the tuyere region of the furnace to offset the reduction in the furnace coke rate. The sulfur content of this coal is $.78 \%$ and is considered to be mid-range. Candidate coals that were evaluated for use ranged in sulfur content from $.32 \%$ to $1.75 \%$. The sulfur content and the impact on the furnace process are discussed in more detail later. The sizing of the final granulated coal product is also important to the blast furnace operators. Daily samples are taken on each furnace to determine the size distribution of the coal sent to the furnace. Table 2 shows the average size distribution of the coal injected on $\mathrm{C}$ furnace for October. Granular coal size for injection purposes is defined as $100 \%$ of the product coal passing a 4 Mesh (5mm) screen, 98\% -7 Mesh (3mm) and 10-30\% as -200 Mesh. In contrast, pulverized coal is defined as $70 \%-80 \%$ of the product coal -200 Mesh. The definition of granular coal on $\mathrm{C}$ furnace for October is met with the average values shown on Table 2.

The injected coal rate of 264 pounds/NTHM on C furnace during October is one of the highest achieved since the start-up of the coal facility. The reliability of the coal system enabled the operators to reduce furnace coke to a low rate of 661 pounds/NTHM. The low coke rate is not only good economically, it is an indicator of the efficiency of the furnace operation with regard to displacing coke with injected coal.

Hot metal chemistry, particularly silicon and sulfur content, is another important ironmaking parameter. The end user of the molten iron, the Steelmaking Department, specifies the silicon and sulfur levels that are acceptable for their process. Low variability around the average value is necessary to achieve these specifications. The standard deviations of the silicon and sulfur content of the hot metal for October are shown on Table 1.

Table 1 also shows a typical period of natural gas injection on the $\mathrm{C}$ furnace during January 1995. Comparatively, we can see the significant operating changes that occur with the use of injected coal versus natural gas. The wind volume on the furnace has decreased significantly with the use of coal. Oxygen enrichment also increased from $24.4 \%$ to $27.3 \%$ with coal. The amount of moisture added to the furnace in the form of steam increased most significantly from 3.7 grains/SCF of wind to 19.8 grains/SCF. All of these operating variables were increased by the furnace operating personnel to maintain adequate burden material movement. These actions also increased the permeability of the furnace burden column. Permeability is discussed in more detail later. 
Also of significance in Table 1 is the adjustment made to the furnace slag chemistry to accommodate the increased sulfur load from the injected coal. The sulfur content of the slag increased from $0.85 \%$ with gas to $1.39 \%$ with coal. The slag volume also increased in order to help with the additional sulfur input.

Blast furnace slag chemistry and volume is a determining factor in the final sulfur content in the hot metal. The blast furnace slag must be of such a chemistry that it can carry the sulfur supplied by the burden material, including the sulfur contributed by the injected coal. Table 3 shows the sulfur balance on $\mathrm{C}$ furnace during the month of October. Injected coal is the second largest contributor of sulfur to the blast furnace process. The blast furnace slag is the largest output variable for the sulfur.

The blast furnace also produces large quantities of gas. The gas exits the top of the furnace, is cleaned and used as a fuel in the hot blast stoves. The excess gas produced is consumed at the plant's boiler house. Special testing during October by the Burns Harbor Plant

Environmental Department for the presence of sulfur in the gas shows an average of 3.1 grains per 100 scf during the month. The amount of sulfur present in the gas and the total gas production is shown on Table 3. The total furnace sulfur balance shows reconciliation of the furnace sulfur input to output at $99.2 \%$.

A method of representing furnace stack conditions as well as the overall furnace operation is by calculating a permeability value. Permeability is a function of the blast rate and the pressure drop through the furnace. The equation used for this purpose is:

Permeability $=(\text { Furnace Wind Rate })^{2} /\left[(\text { Furnace Blast Pressure })^{2}-(\text { Furnace Top Pressure })^{2}\right]$

The larger the permeability number the better the furnace burden movement and the better the reducing gas flows through the furnace column. Figure 1 is a plot of the permeability value and the injected coal rate for each month in 1996. The permeability decreased from January to February as the injected coal rate was increased. Since then, this value has increased monthly, declining only slightly to a level of 1.19 for October. This indicates an acceptable overall operation on the $\mathrm{C}$ furnace during the base test period.

\section{ENVIRONMENTAL TEST RESULTS}

\section{Gaseous Streams:}

During the month weekly gas samples were obtained from the $\mathrm{C}$ furnace and analyzed by Mostardi Platt Associates, Inc. Results of the gas samples are presented in Appendix 1. 
Wastewater Monitoring

During October, monitoring of the Division's treated process water effluent (Monitoring Station 011) and the Division's combined effluent was conducted in accordance with the NPDES permit. In addition, internal monitoring of the Blast Furnace Recirculating Water System was performed weekly. Figure 2 is a flow diagram of the water system and shows the location of the outfall monitoring stations. All monitoring results at Station 011 and Outfall 001 were within the applicable limitations and/or expected ranges. Monitoring results for the recirculating water system on October 23 indicate a slightly elevated ammonia-nitrogen concentration. The cause of elevated level is unknown. There were no adverse affects on the Division's wastewater system that could be attributed to the BFGCI system during the month. Appendix 2 shows the monitoring results for the month.

\section{FURNACE THERMAL CONDITIONS AND LINING WEAR}

The $\mathrm{C}$ furnace is equipped with a Thermal Monitor System consisting of two components: eight thermocouples embedded in the furnace refractory at each of four furnace elevations and an extensive system of thermocouples in the discharge water cooling system at five furnace elevations. The heat loss in the furnace is calculated for various elevations in the furnace from the water system thermocouples.

In addition to the array of thermocouples, wear monitors have been placed in the refractories of the furnace at various elevations and quadrants. These monitors give an indication of the amount of brick that is left in the furnace at the various elevations.

The inwall refractory temperatures for $\mathrm{C}$ furnace are shown in Figure 3 for 1996. The increased amount of injected coal does not appear to have caused an increase in the temperatures over the ten month time frame. The refractory temperatures for October have decreased at several elevations from some high values during January and February.

By contrast, the thermal load values in BTU/HR/FT' ${ }^{2}$ especially at cooler plate row $11-20$, did increase significantly during May, June and July compared to January 1996. However, the heat loads have subsided during the following three months. This trend is shown in Figure 4. Although there has been a increase in thermal loads at row 11-20, the mid-stack elevation on the furnace, none of the other measured elevations have increased significantly. Changes in thermal load values indicate a change in the operating characteristics of the furnace. The $\mathrm{C}$ furnace, as mentioned previously, was designed to accept these anticipated increases.

Figure 5 shows the refractory wear monitor readings from the beginning of the $\mathrm{C}$ furnace campaign. The amount of coal injected is also shown. This figure seems to indicate that brick wear has increased as coal injection rates have been increased. This may or may not be the proper conclusion on furnace refractory wear. Figure 6 is included in this analysis to 
show that refractory wear in a blast furnace may also be attributed to normal wear over the life of the campaign. Figure 6 shows the refractory wear patterns of previous furnace campaigns at the Burns Harbor Plant against service time in months. We note that after twenty months of service, the highest wear area on $C$ furnace with coal injection is slightly better than three previous furnace campaigns without coal injection. We must also note, however, that the previous campaigns shown did not utilize the high density cooling configuration that was installed on the $\mathrm{C}$ furnace for the current campaign. More operating data is necessary to determine the relationship between coal injection and furnace refractory wear.

\section{DISCUSSION}

A major conclusion of the use of granular coal injection for the October base test as well as the general furnace operational characteristics shown throughout 1996 is that granular coal performs very well in large blast furnaces.

The quantity of furnace coke that is replaced by an injected fuel is an important aspect of the overall value of the injectant on the blast furnace process. The replacement ratio is also a very strong indication of the quality of the overall operation with coal as the injectant. A detailed analysis of the furnace coke/granulated coal replacement value for the $C$ and $D$ furnaces at the Burns Harbor Plant has been completed.

The replacement ratio for a blast furnace injected fuel is defined as the amount of furnace coke/NTHM that is replaced by one pound/NTHM of the injectant. However, there are many furnace operating factors, in addition to the injectant, that affect the reported coke rate. In order to calculate an accurate value for the injected coal's role in the process, all other blast furnace operating variables that result in coke rate changes, positively or negatively, must be accounted for. After technically accounting for coke changes caused by variables other than the coal, we attribute the remaining coke difference to the injected coal.

This evaluation uses monthly average furnace operating results compared to an appropriate base period for each furnace to develop the replacement ratio. We have used twenty five months of data on both furnaces which includes operating results through the second quarter of 1996. The more monthly operating data available the more accurate and appropriate the replacement value determination will be.

The adjusted furnace coke rate and the injected coal are plotted in Figure 7 along with the best fit regression line. The slope of the best fit line shows that the coal/coke replacement is 0.96. The $C$ furnace value for October 1996 is shown seperately. This value correlates well with the overall regression. This is an excellent replacement ratio and is significantly better than the 0.8-0.9 replacements reported by other injection operations. 
A second conclusion from this work is the ability of the process to adequately handle the increased sulfur loading from the injected coal. As shown in the sulfur balances, the blast furnace slag can be adjusted, without harm to the overall operation, to accommodate the increased sulfur input.

Thirdly, the unexpectedly large decrease in furnace permeability as a result of the use of injected coal has been overcome by increasing the oxygen enrichment and raising the moisture additions to the furnace. 
TABLE 1

\section{BASE PERIOD EVALUATION \\ Burns Harbor C Furnace \\ Summary of Operations}

\section{OCTOBER 1996}

Production, NTHMday

Delays, Min/day

Coke Rate, Ib/NTHM Rep.

Natural Gas Rate, Ibs/NTHM

Injected Coal Rate, Ibs/NTHM

Total Fuel Rate, Ibs/NTHM

\section{Burden \%:}

Sinter

Pellets

Misc.

BOF Slag Ibs/NTHM

Blast Conditions:

Dry Air SCFM

Blast Pressure, psig

Permeability

Oxygen in Wind \%

Temp, $\mathbf{F}$

Moist. Grs/SCF

Flame Temp, $F$

Top Temp, F

Top Press, psig

Coke:

$\mathrm{H} 2 \mathrm{O}, \%$

Hot Metal \%:

Silicon

Standard Dev.

Sulfur

Standard Dev.

Phos.

Mn.

Temp., $F$

Slag \%:

$\mathrm{SiO} 2$

$\mathrm{Al} 2 \mathrm{O} 3$

$\mathrm{CaO}$

$\mathrm{MgO}$

$\mathrm{Mn}$

Sulfur

$B / A$

B/S

Volume, Ibs/NTHM
6943

71

661

0

264

925

35.9

63.8

.3

5

137,005

38.8

1.19

27.3

2067

19.8

3841

226

16.9

5.0

.50

.128

.040

.014

.072

.43

2734

36.54

9.63

39.03

11.62

.46

1.39

1.10

1.39

424
4.8

JANUARY 1995

7436

25

740

141

0

881

32.3

67.0

.7

0

167,381

38.9

1.57

24.4

2067

3.7

3620

263

16.1

.44

.091

.043

.012

.070

.40

2745

38.02

8.82

37.28

12.02

.45

0.85

1.05

1.30

394 


\section{TABLE 2}

\section{BURNS HARBOR C FURNACE INJECTED COAL ANALYSIS AND SIZING OCTOBER 1996 - COAL TEST BASE}

Coal

Vol. Matter, \%

Sulfur, \%

Ash, \%

Ultimate Analysis, \%

Carbon

Oxygen

Hydrogen

Nitrogen

Chlorine

Total Mois.,\%

GHV, BTU/lb (dry)
Va. Pocahantas

Six Train Avg. , June1996

18.00

.78

5.3

87.1

1.23

4.2

1.21

.170

6.6

14974
C FURNACE PRODUCT COAL SIZING

OCTOBER 1996

MEAN \%

0

0.6

0.2

3.7

0.5

-8 Mesh +16 Mesh

10.6

1.1

16.0

0.6

26.8

4.6

27.7

4.2

13.9

3.3

-200 Mesh +325 Mesh

-325 Mesh

TOTAL $\frac{0.70}{100.0}$ 
TABLE 3

\section{BURNS HARBOR C FURNACE SULFUR BALANCE OCTOBER 1996 - COAL TEST BASE}

\section{SULFUR INPUT:}

Material;

Furnace Coke, Sulfur Analysis Tons Coke Used

Tons Sulfur In

Injected Coal,Sulfur Analysis

Tons Coal Used

Tons Sulfur In

Sinter, Sulfur Analysis

Tons Sinter Used

Tons Sulfur In

Pellets,Sulfur Analysis

Tons Pellets Used

Tons Sulfur in

Scrap,Sulfur Analysis

Tons Scrap Used

Tons Sulfur in

BOF Slag, Sulfur Analysis

Tons BOF Used

Tons Sulfur in

TOTAL TONS Of SULFUR IN:
October 1996

$71,085.0$

490.5

$.78 \%$

$28,409.0$

221.6

$.02 \%$

$121,282.6$

24.3

$.01 \%$

$215,306.5$

21.5

$.23 \%$

$3,981.7$

9.2

$.07 \%$

530.2

.4

767.5
SULFUR OUTPUT:

Material;

Blast Furnace Slag, Sulfur Analysis

Total Tons Produced

Tons Sulfur Out

Blast Furnace Iron,Sulfur Analysis

Total Tons Produced

Tons Sulfur Out

Fiue Dust, Sulfur Analysis

Total Tons Produced

Tons Sulfur Out

Filter Cake,Sulfur Analysis

Total Tons Produced

Tons Sulfur Out

Top Gas, Sulfur Content

Total Gas Produced, MMCF

Tons Sulfur Out
October 1996

$1.39 \%$

$45,626.6$

634.2

$.040 \%$

$215,220.0$

86.1

$.450 \%$

$1,076.1$

4.8

$.482 \%$

$2,570.60$

12.4

3.1 Grs./100 scf 108,246

23.9
TOTAL TONS of SULFUR OUT:

761.4 


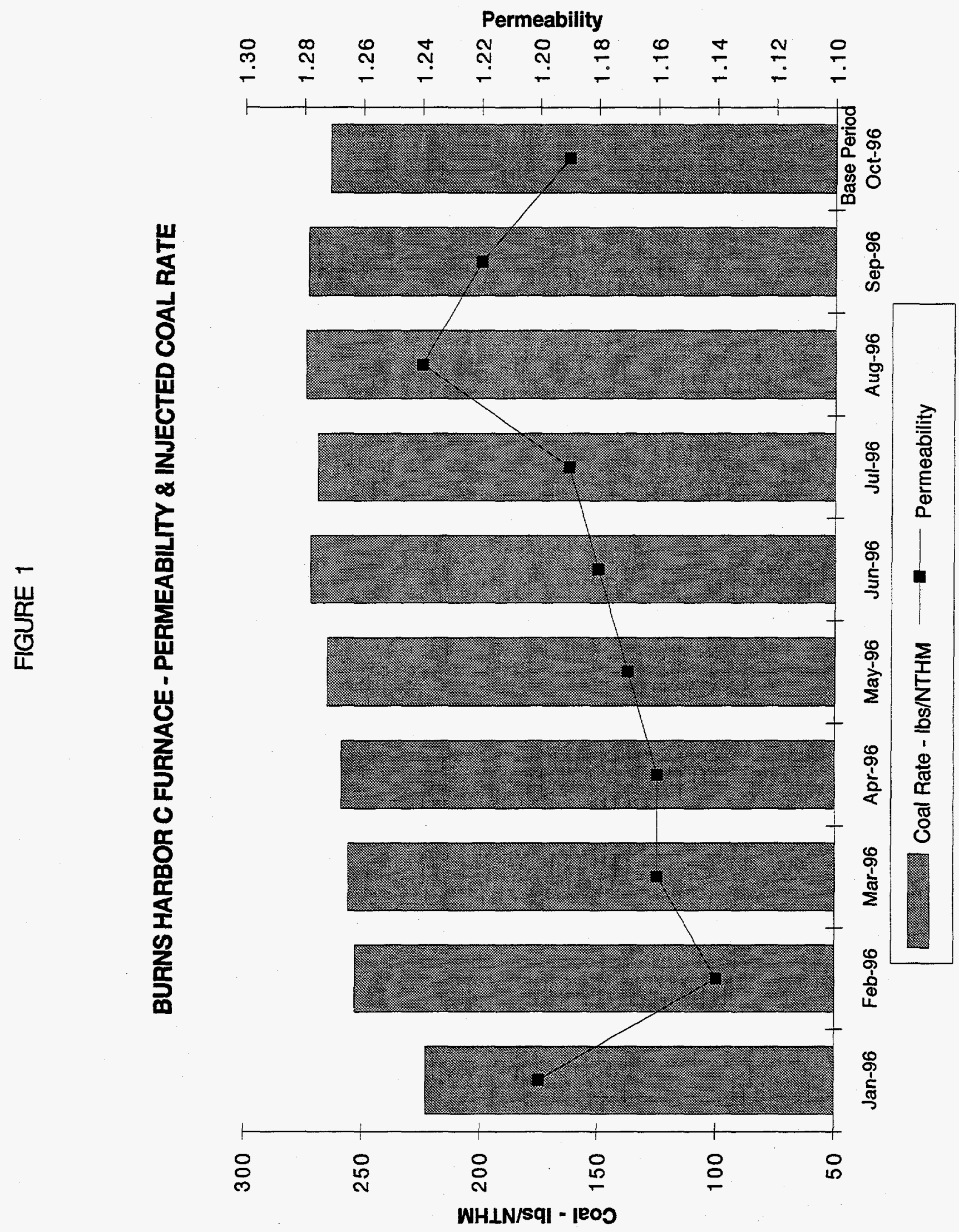



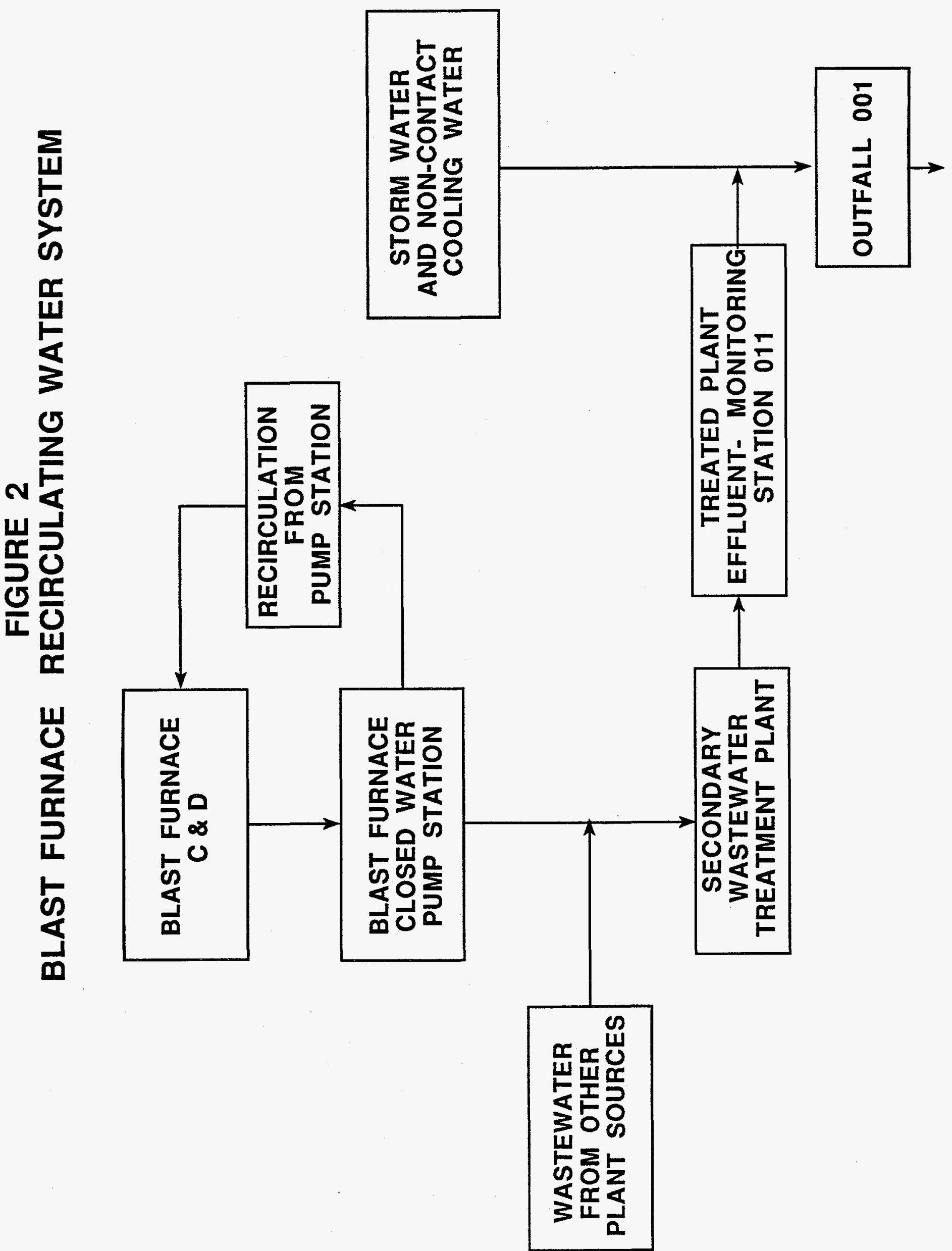


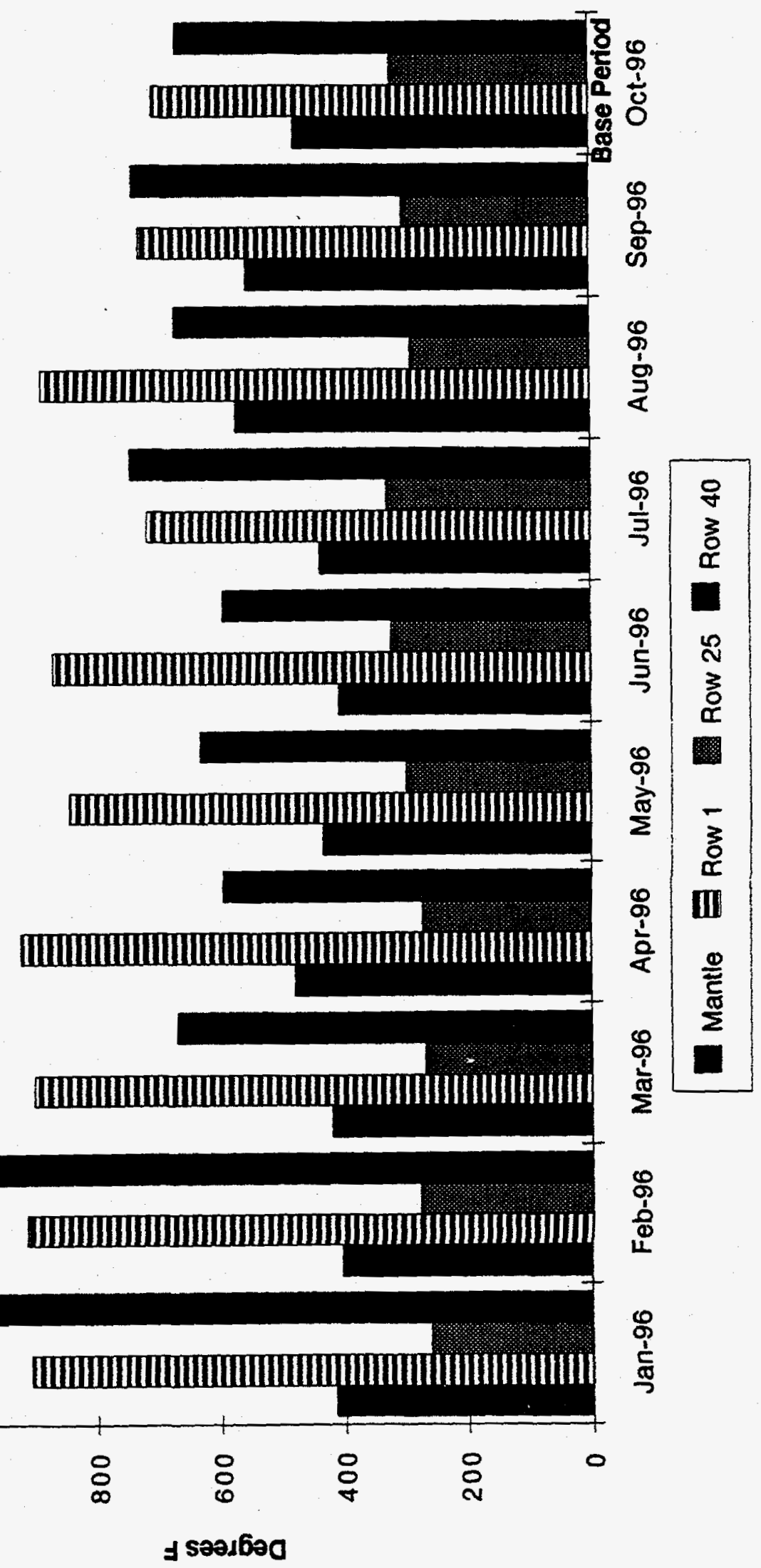




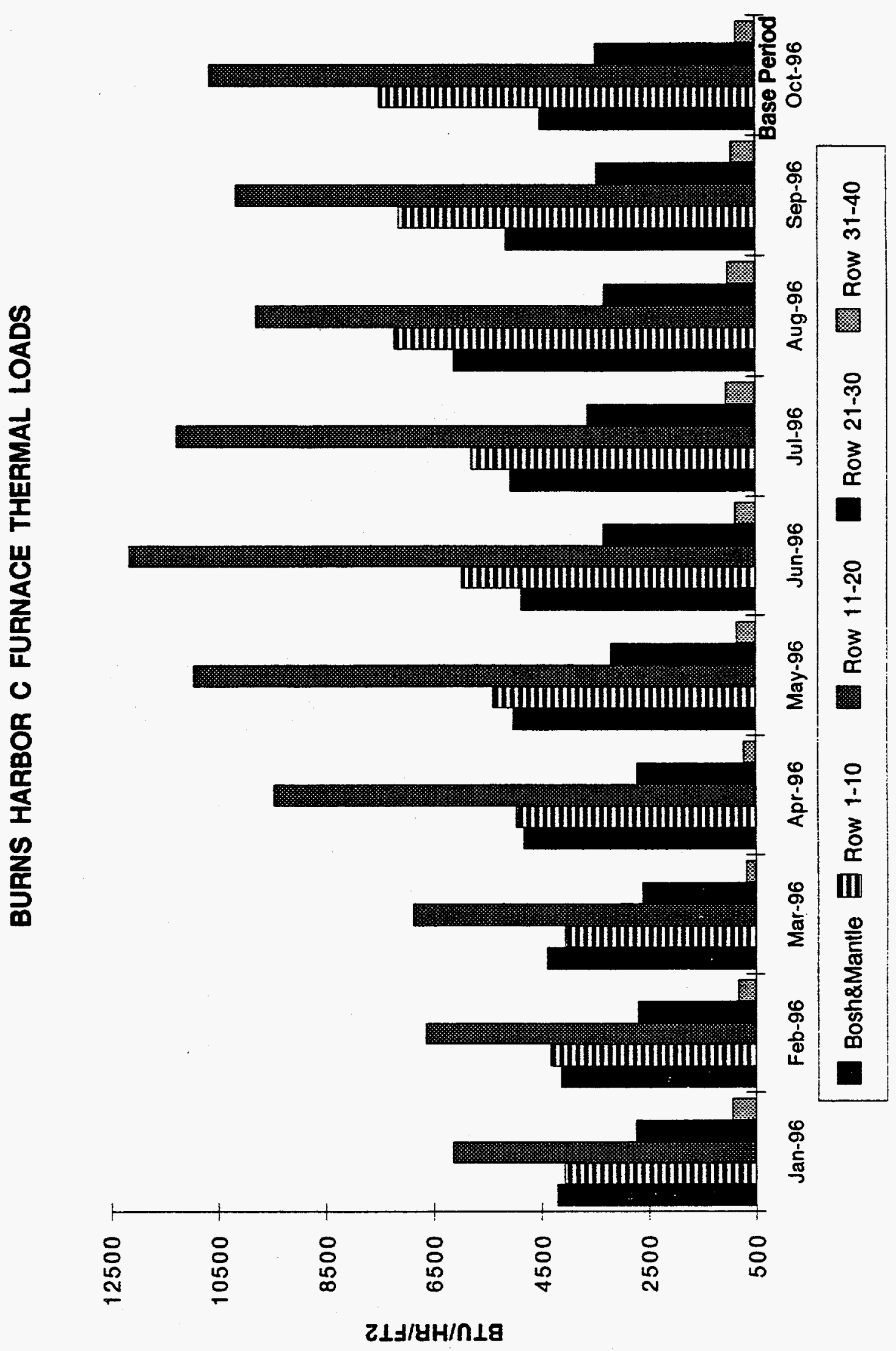



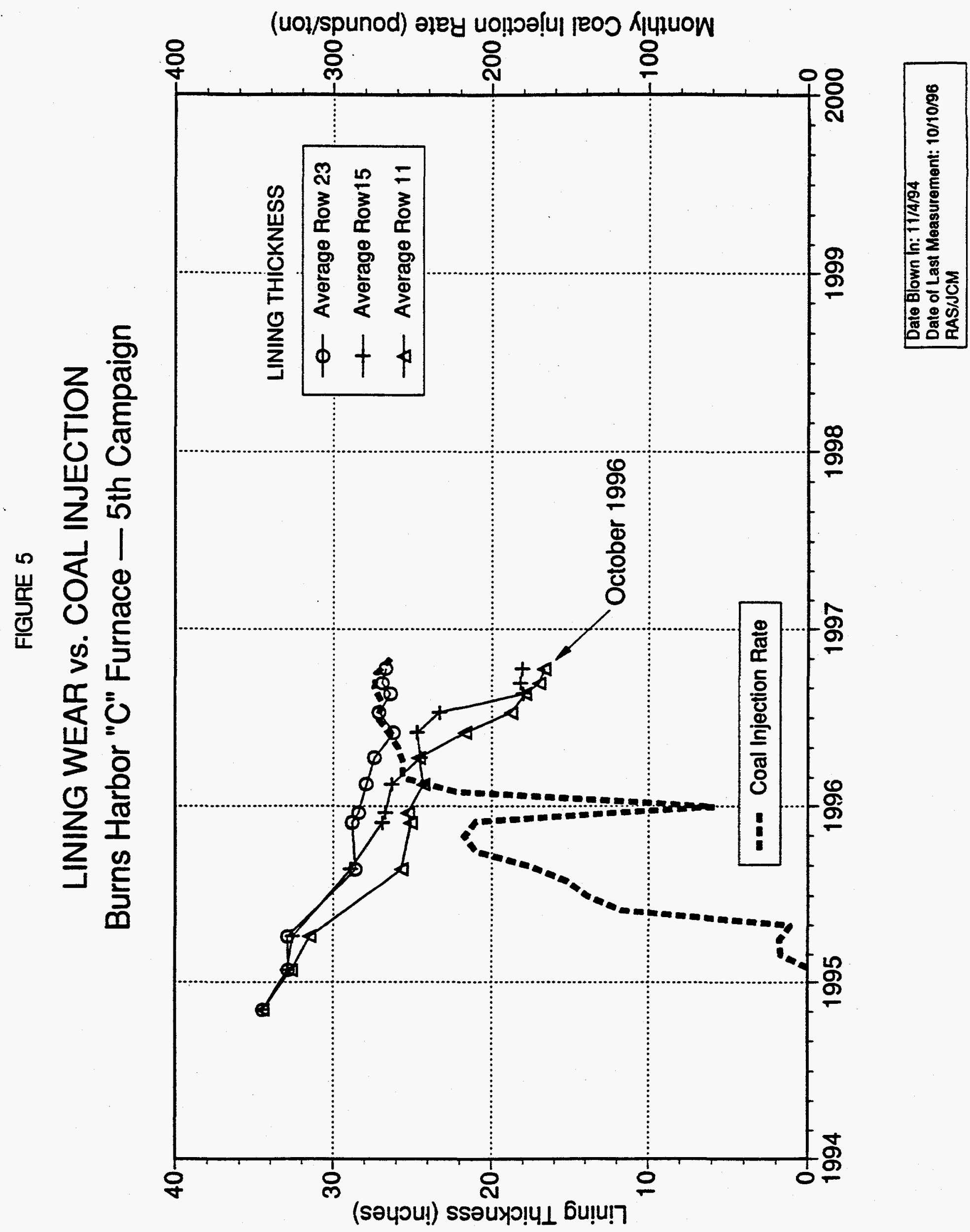


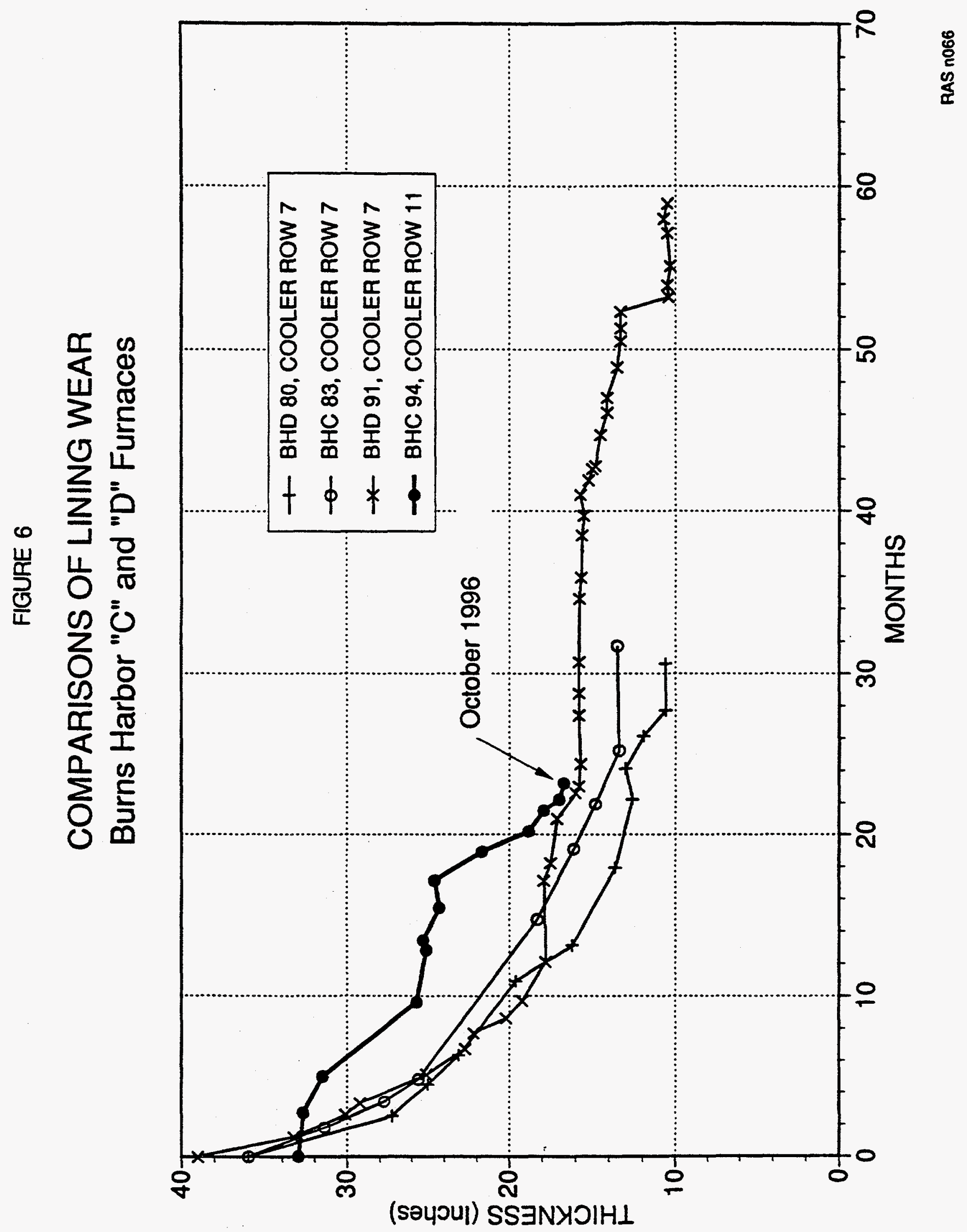




\section{FIGURE 7}

\section{BURNS HARBOR C \& D BLAST FURNACES}

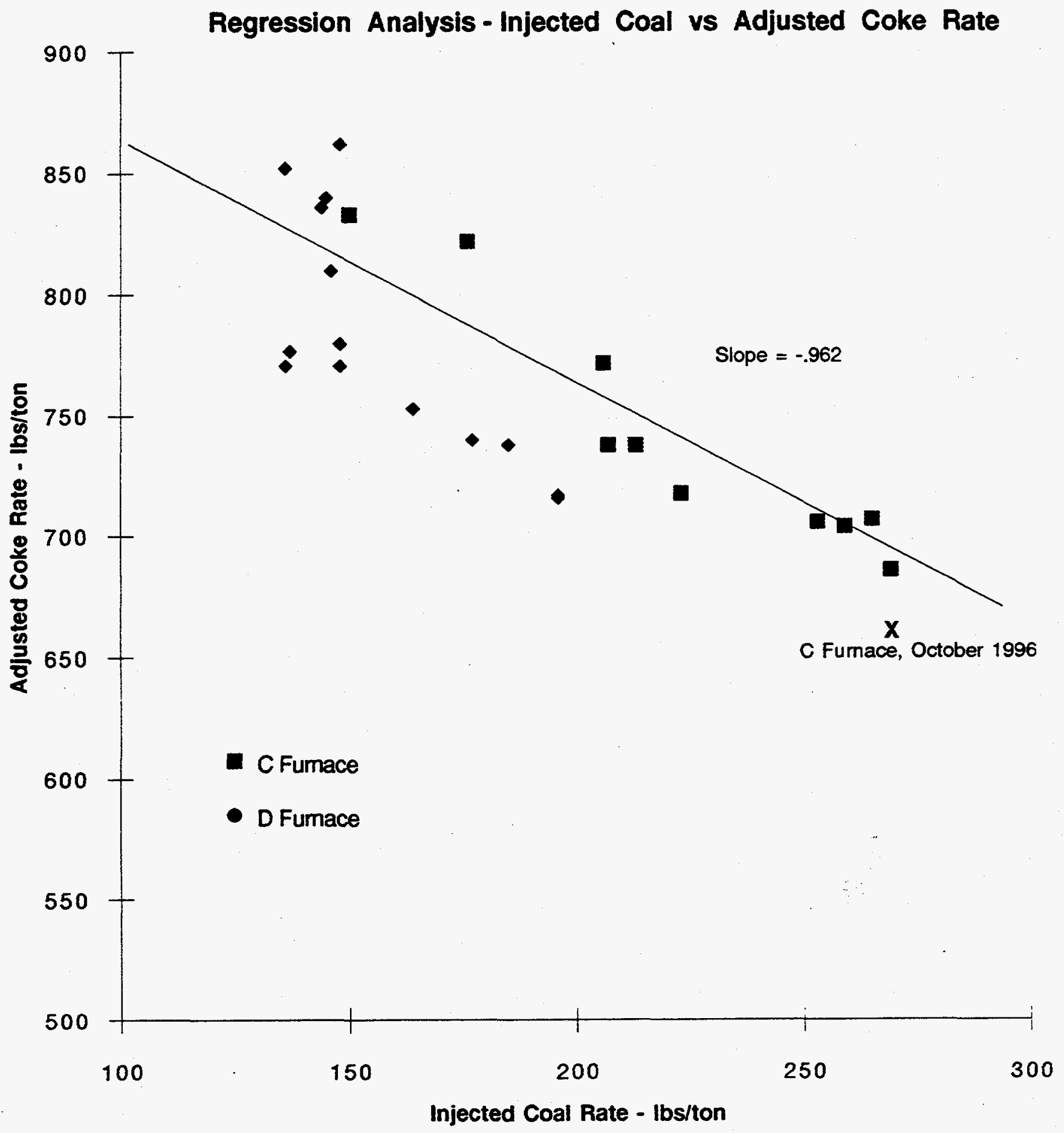


Blast Furnace Granulated Coal Injection

Environmental Monitoring Report

Appendix I - Gaseous Stream Testing Results 


\title{
$\Rightarrow$ Mostardi Platt
}

\author{
GAS ANALYSIS STUDY \\ Performed For \\ BETHLEHEM STEEL CORPORATION \\ At The \\ Burns Harbor Works \\ Burns Harbor, Indiana \\ Blast Furnace $\mathbf{C}$ \\ October 3, 9, 17 and 25, 1996
}

- Copyright 1996

All rights reserved in

Mostardi-Platt Associates, Inc.

MOSTARDI PLATT PROJECT: $64017,64113,64202,64312$

DATE SUBMITTED: NOVEMBER 6, 1996 
Bethlehem Steel

C-Blast Furnace

Gas Test Results

Burns Harbor, Indiana

\begin{tabular}{|c|c|c|c|c|c|c|c|}
\hline \multirow[b]{2}{*}{$\begin{array}{c}\text { Date } \\
\text { Sampled }\end{array}$} & \multirow[b]{2}{*}{$\begin{array}{c}\text { Time } \\
\text { Sampled }\end{array}$} & \multirow[b]{2}{*}{$\begin{array}{c}\text { Hydrogen } \\
\text { (mol \%) }\end{array}$} & \multirow[b]{2}{*}{$\begin{array}{c}\mathrm{CO}_{2} \\
(\mathrm{~mol} \%)\end{array}$} & \multirow[b]{2}{*}{$\begin{array}{c}\mathrm{O}_{2} \\
(\mathrm{~mol} \%)\end{array}$} & \multirow{2}{*}{$\begin{array}{c}\text { Carbon } \\
\text { Monoxide } \\
\text { (mol \%) }\end{array}$} & \multicolumn{2}{|c|}{ Total Sulfur Content } \\
\hline & & & & & & (ppmv) & (2s gr/100 sct) \\
\hline $10 / 03 / 96$ & 0830 & $*$ & - & * & $*$ & - & * \\
\hline $10 / 03 / 96$ & 1100 & 4.58 & 25.0 & 0.63 & 23.7 & 36.0 & 2.3 \\
\hline $10 / 03 / 96$ & 1300 & $*$ & $*$ & $*$ & * & - &. \\
\hline $10 / 09 / 96$ & 0800 & 2.26 & 21.8 & 3.99 & 21.3 & 34.0 & 2.1 \\
\hline $10 / 09 / 96$ & 1100 & * & * & $*$ & $*$ & * & * \\
\hline $10 / 09 / 96$ & 1300 & 4.04 & 23.6 & 1.26 & 24.2 & 60.0 & 3.8 \\
\hline $10 / 17 / 96$ & 0800 & * & * & * & * & * & $*$ \\
\hline $10 / 17 / 96$ & 1100 & $*$ & $*$ & $*$ & * & $*$ & $*$ \\
\hline $10 / 17 / 96$ & 1300 & 4.73 & 24.9 & 0.62 & 25.5 & 49.0 & 3.1 \\
\hline $10 / 25 / 96$ & 0800 & 4.51 & 24.1 & 0.84 & 24.8 & 59.0 & 3.7 \\
\hline $10 / 25 / 96$ & 1000 & 4.66 & 24.5 & 0.61 & 25.8 & 53.0 & 3.3 \\
\hline $10 / 25 / 96$ & 1200 & 4.49 & 24.3 & 0.94 & 24.5 & 57.0 & 3.6 \\
\hline
\end{tabular}

* Data included in report but shows high \% of oxygen and may not be representative of acrual conditions. 


\begin{tabular}{|c|c|c|c|}
\hline \multicolumn{4}{|c|}{ Major Component Gas Analysis By Gas Chromatoeraphy } \\
\hline \multicolumn{4}{|c|}{ Cient Nere: Mosturdi Plant } \\
\hline \multicolumn{4}{|c|}{ IGT Sample Nuraber. 9614962} \\
\hline Sample Deceription: & Semple 64017-002 & $10 / 3 / 96$ & $11 \cdot 00$ \\
\hline \multicolumn{2}{|c|}{ Date Anabyed: 7-0at-66 } & \multicolumn{2}{|l|}{ Anabet da } \\
\hline Component & Mat $\%$ & Det unat & Welate $\%$ \\
\hline Hedim & $N$ & $0.001 \%$ & ND \\
\hline Hatrow & seste & $0.05 \%$ & esere \\
\hline Croso Dionide & $250 \%$ & $0.03 x$ & $35.7 \%$ \\
\hline Estax & & $0.005 \times$ & \\
\hline Eth-e & & $0.03 \times$ & \\
\hline$a x p=0 / A y=x$ & .00 & $0.03 \times$ & $.07 x$ \\
\hline Nisoper & $\mathbf{4 5 . 1 \times}$ & $0.03 x$ & 61.9\% \\
\hline Methe & & $0.03 *$ & \\
\hline Crtos Manowite & $23.7 \times$ & $0.03 \%$ & 215\% \\
\hline Evtree & & $0.002 x$ & \\
\hline Propene & & $0.002 x$ & \\
\hline Propene & & $0.003 \times$ & \\
\hline Fropadiox & & $0.002 x$ & \\
\hline Propye & & $0.000 x$ & \\
\hline i-Buex & & $0.0002 x$ & \\
\hline DDanere & & $0.002 x$ & \\
\hline 1. Buesce & & $0.002 x$ & \\
\hline i-Bunax & & $0.002 \times$ & \\
\hline Tre-2-Buese & & $0.002 x$ & \\
\hline Cis-2-Buex & & $0.002 x$ & \\
\hline 13-Bundiene & & $0.002 x$ & \\
\hline Do-Penter & & $0.001 \%$ & \\
\hline ipterene & & $0.002 x$ & \\
\hline DPenerex & & $0.002 x$ & \\
\hline Penenos & & $0.002 x$ & \\
\hline$H=0 \times$ Phis & & $0.002 x$ & \\
\hline Hydrow Suriade & & $0.0001 \%$ & \\
\hline Corbangl Sutride & $.0036 \%$ & $0.0001 \%$ & $.0071 \%$ \\
\hline Unidenifred & & $0.001 \%$ & \\
\hline Wea & No & $0.001 \%$ & No \\
\hline Tad & $100.0 \%$ & & $100.0 \%$ \\
\hline Colendexed $R$ & iol Ger Propertien per 1 & 1 Diste-si & \\
\hline$\tau \operatorname{cosp}(7)$ & $-\cos$ & 60 & \\
\hline Prom (pris) & IKas & $1<73$ & \\
\hline Comprembiling Fan $[2]=$ & 0.95900 & 0.99900 & \\
\hline Reloive Dasing = & 1.0657 & 1.0657 & \\
\hline GNo HV (ORY) . & 91.6 & 91.8 & \\
\hline Gow HN (SAT.) & 90.0 & 902 & \\
\hline Wabibe bodar = & 88.3 & 89.0 & \\
\hline$N=F V(D r y)=$ & 82.5 & ๓.7 & \\
\hline Net frV (SAC) = & 37.0 & 87.2 & \\
\hline \multicolumn{4}{|c|}{ 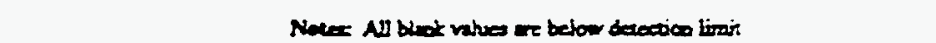 } \\
\hline & No. Na Dentions & $0.03 \%$ & \\
\hline
\end{tabular}




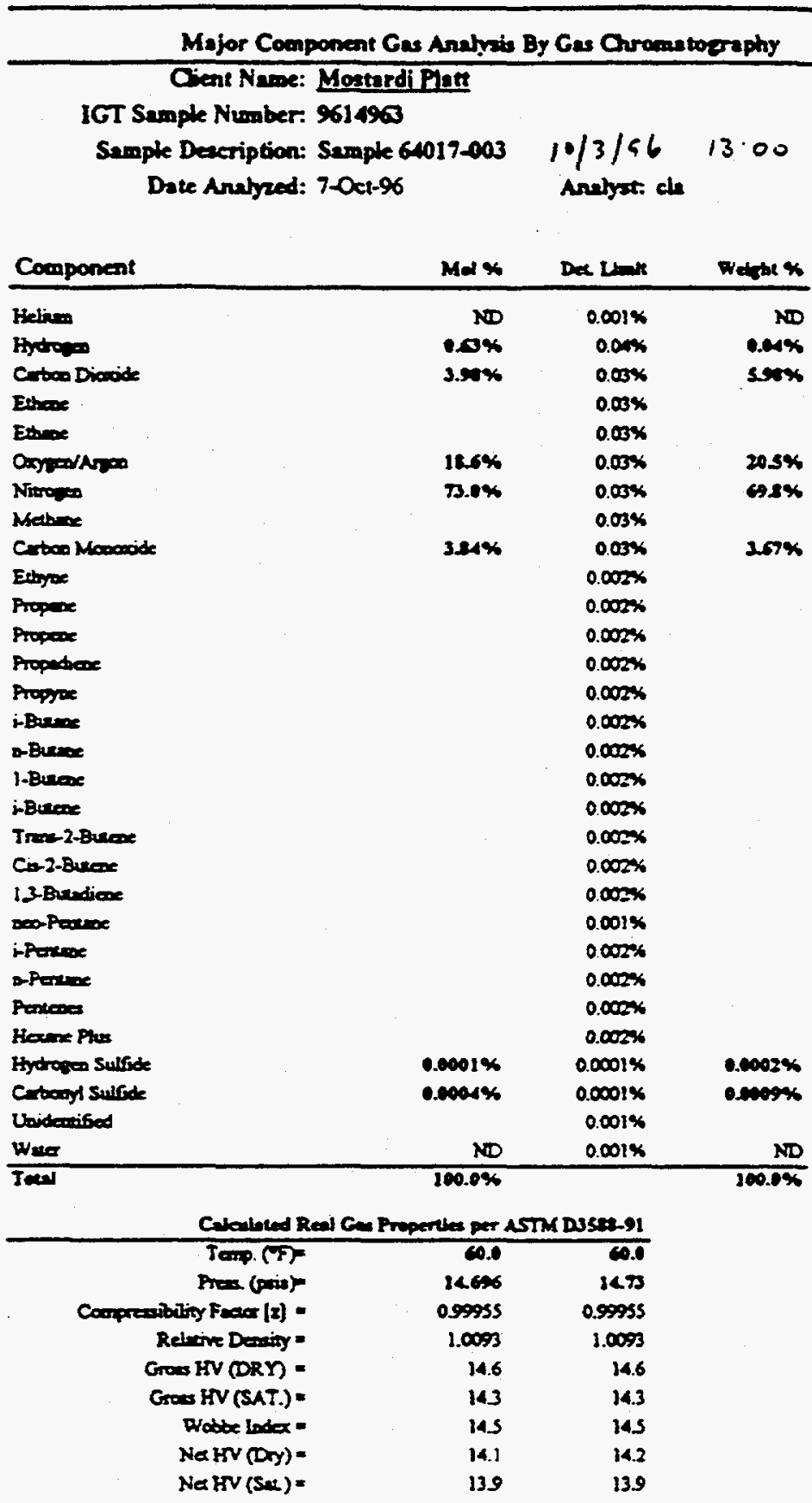

Niver. Nil bient vitues are below decection bonst ND. Na. Datermed

Ial tydroubars be beloo $0.03 \%$ 


\begin{tabular}{|c|c|c|c|}
\hline \multicolumn{4}{|c|}{ Major Component Ges Anabysis By Gas Chromsto eraphy } \\
\hline $\begin{array}{l}\text { Cient Nawe: } \\
\text { IGT Sample Number: } \\
\text { Sample Decripton: } \\
\text { Date Anabred: }\end{array}$ & Piatt & $\begin{array}{l}10 / 9 / 96 \\
\text { Andyar: do }\end{array}$ & 0800 \\
\hline Compoment & Max $\%$ & Dec umat & Wright $\%$ \\
\hline Heliven & n & $0.01 \%$ & ND \\
\hline Hatrex & $226 \times 6$ & $\operatorname{aons}$ & $0.15 \%$ \\
\hline Citoon Diciode & $225 \%$ & a.es\% & 3024 \\
\hline Ethene & & acses & \\
\hline Estax & & Q.03\% & \\
\hline$a_{y y=0}$ & $399 \times$ & 20036 & $\$ 15 \%$ \\
\hline Nixom & $50.7 \times$ & $\alpha \cos x$ & $\$ 4.7 \%$ \\
\hline Mether & & $0.03 \%$ & \\
\hline Carboo Noopode & $213 \times$ & $0.03 *$ & $192 \%$ \\
\hline Estoree & & $0.000 x$ & \\
\hline Propese & & $0.002 x$ & \\
\hline Propene & & $0 . \cos x$ & \\
\hline Propectione & & $0.002 x$ & \\
\hline Propye & & $0.002 x$ & \\
\hline iberex & & $0,000 x$ & \\
\hline De & & $0.002 x$ & \\
\hline 1-buene & & $0.002 * 6$ & \\
\hline ibrecox & & $0.002 x$ & \\
\hline Iras-2-Burex & & $0.002 \times 6$ & \\
\hline Cir 2 -beace & & 0.0006 & \\
\hline 1.3-Bundiere & & $2 \cos x$ & \\
\hline$=00$ Pentone & & $2001 \%$ & \\
\hline iprentere & & $0.002 \times 6$ & \\
\hline oporime & & $0.002 x$ & \\
\hline Perenes. & & $0.002 \times 6$ & \\
\hline Heoc Phin & & $0.002 x$ & \\
\hline Hychepen Sulficts & & $00001 \%$ & \\
\hline Curboyl Sulfde & $0.0034 \%$ & $0.0001 \%$ & 0.00034 \\
\hline Uniderified & & $0.001 \%$ & \\
\hline Wure & ND & $0.001 \%$ & ND \\
\hline Ined & 1020 & & 100.04 \\
\hline Crenined R & perisent $A$ & (Dsseats & \\
\hline Tapp (T) & $\infty$ & 20 & \\
\hline Pren (pin) & 1400 & $14 \pi$ & \\
\hline 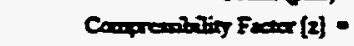 & 059007 & 0959007 & \\
\hline Redeive Desing = & 1.0731 & 1.0731 & \\
\hline GOAV (DRY) - & 759 & 76.1 & \\
\hline Gros FV (SAT.) & 38.6 & 20.8 & \\
\hline Woble lodex . & 33 & is. & \\
\hline $\mathrm{NE} \operatorname{sV}(\mathrm{Dry})=$ & 24,4 & 24.6 & \\
\hline $\mathrm{Ne} \operatorname{HV}(\mathrm{S}-\mathrm{x})=$ & 73.1 & 33 & \\
\hline Riner & 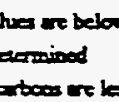 & 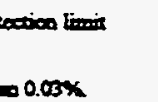 & \\
\hline
\end{tabular}




\begin{tabular}{|c|c|c|c|}
\hline \multicolumn{4}{|c|}{ Major Component Gas Arelysis By Gas Chromatography } \\
\hline \multicolumn{4}{|c|}{ Cient Nance: Mostandi Platt } \\
\hline \multicolumn{4}{|c|}{ IGT Serpie Number: 9615122} \\
\hline Sumple Deacripdon: & Sampie 64113-002 & $10 / 9 / 96$ & $11: 00$ \\
\hline \multicolumn{2}{|c|}{ Date Anabyed: $23-0 \mathrm{ct}-96$} & \multicolumn{2}{|l|}{ Arabste ele } \\
\hline Comproment & Mar $\times$ & Der Limin & Wedtex $x$ \\
\hline Hediven & N & $a, \infty 1 \%$ & No \\
\hline Fyprom & e.735 & eas & a.es\% \\
\hline Citroe Dionode & $3.75 \%$ & $0.00 \%$ & $\operatorname{sen} x$ \\
\hline Estexe & & a.03x & \\
\hline Estare & & $0.03 \times$ & \\
\hline anyodingeo & $18.6 \%$ & $0.03 \times$ & 2034 \\
\hline Niropen & $7.1 \times$ & $0.03 \%$ & $70.0 \%$ \\
\hline Wehore & & $0.05 \%$ & \\
\hline Crateon Monosode & $390 \%$ & $0.003 \%$ & 1.74\% \\
\hline Etrope & & $0.000 \times$ & \\
\hline Propore & & $0.002 x$ & \\
\hline Propene & & $0.002 x$ & \\
\hline Propadiene & & $0.002 \%$ & \\
\hline Propres & & $0.000 \times$ & \\
\hline ibuaroe & & $a \cos x$ & \\
\hline a-Burne & & $0.002 \times$ & \\
\hline 1-Buese & & $0.002 x$ & \\
\hline ibuene & & $0.002 x$ & \\
\hline Tres-2-Buexe & & $0.000 \times$ & \\
\hline Cin-28enese & & $0.002 x$ & \\
\hline 13-Buratiene & & $0.002 x$ & \\
\hline Deop Pentex & & $a, 001 \%$ & \\
\hline Fanese & & $0.000 \times$ & \\
\hline DPanes & & $0.002 x$ & \\
\hline Penenge & & $0.002 x$ & \\
\hline Fecose Phas & & $20002 \%$ & \\
\hline Hytroges Sulite & $.0001 \%$ & $00001 \%$ & $0.0001 \%$ \\
\hline 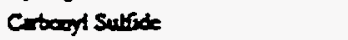 & e.mex & $20001 x$ & $.00000 x$ \\
\hline Uaderaified & & $0.001 \times$ & \\
\hline wer & ND & $2001 x$ & ND \\
\hline Tom & $\tan x$ & & $100.0 \times$ \\
\hline \multicolumn{4}{|c|}{ 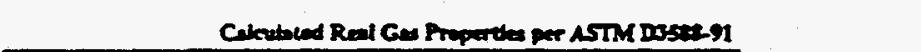 } \\
\hline $\operatorname{Tam}(9)$ & $\cos$ & 6 & \\
\hline pren (pis) & reas & 347 & \\
\hline Comprombing Feer $[2]$ - & 0.99955 & osesss & \\
\hline Relowe Deminy = & $1.00 n$ & $1.00 n$ & \\
\hline$G=H V(D R Y)=$ & 15.1 & 15.I & \\
\hline$G+000$ AV (SAT.) $=$ & 148 & 148 & \\
\hline Wabbe linda = & is.o & 15.0 & \\
\hline$N \in H V(D y)=$ & 14.6 & 14.6 & \\
\hline$N \in \operatorname{sv}(5 x)=$ & 143 & 103 & \\
\hline \multicolumn{4}{|c|}{ 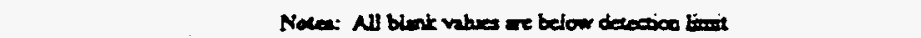 } \\
\hline & ND. Noa Deactived & & \\
\hline & Toul bytrocarbocen ure les & $=0.03 \%$ & \\
\hline
\end{tabular}


Major Component Gas Arelyas By Gas Curometography

Cient Name: Mostandi Plait

IGT Seruple Number: $\$ 615123$

Sample Description: Sample 64113-003

Date Antyred: $23-0 \mathrm{xt} .96$

$10 / 5 / 56 \quad 13.00$

Analyst: de

\begin{tabular}{|c|c|c|c|}
\hline Component & $\operatorname{Mel} \boldsymbol{x}$ & Der Umak & Wrigix $x$ \\
\hline Felium & ND & $0.001 \%$ & : $\mathbf{N D}$ \\
\hline Fydros & 4.4\% & $0.0 \times 16$ & $0.26 \%$ \\
\hline Catur Dioside & $23.6 \%$ & $0.03 \%$ & $33.7 \%$ \\
\hline Eubere & & $0.00 \%$ & \\
\hline Estane & & $0.03 \%$ & \\
\hline 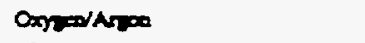 & $126 \%$ & $0.00 \%$ & $132 \%$ \\
\hline Niveren & 2004 & $0.03 \%$ & $27 x$ \\
\hline Metere & & $0.03 \%$ & \\
\hline Cirboe Mononode & $202 \%$ & $0.03 \%$ & 22.46 \\
\hline Eutye & & $0.002 \%$ & \\
\hline Propere & & $0.002 \%$ & \\
\hline Propene & & $0.002 \%$ & \\
\hline Proputiene & & $0.002 \pi$ & \\
\hline Propye & & $0.002 x$ & \\
\hline i-Bueroe & & $0.002 \times$ & \\
\hline arberese & & $0.002 x$ & \\
\hline 1-Benese & & $0.002 \%$ & \\
\hline i-Buecere & & $0.002 \%$ & \\
\hline Tram-2-Burene & & $0.002 *$ & \\
\hline Cin-2-buexe & & $0.002 x$ & \\
\hline 1,3-Buradien & & $0.002 \%$ & \\
\hline nopereres & & $0.001 \%$ & \\
\hline i-Peostare & & $0.002 x$ & \\
\hline apenter & & $0.002 \%$ & \\
\hline Penteres & & $0.002 \%$ & \\
\hline Hoxpe Ptw & & $0.002 x$ & \\
\hline Hydrogen Sutbide & $0.0019 \%$ & $0.0001 \%$ & $0.0021 \%$ \\
\hline Craogt Sulfide & $0.041 \%$ & $0.0001 \%$ & c.max \\
\hline Unidencifised & & $0.001 x$ & \\
\hline Wer & ND & $0.001 \%$ & N \\
\hline Tad & $16.0 \%$ & & $100.0 \%$ \\
\hline \multicolumn{4}{|c|}{ 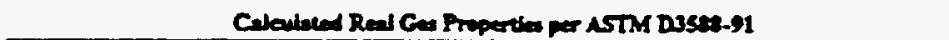 } \\
\hline $\operatorname{Tenp}(\boldsymbol{T})$ & 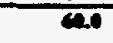 & 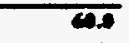 & \\
\hline$P=(p-i))$ & 1003 & 1473 & \\
\hline Cocprenibiting Fead $[2]=$ & 0.90003 & 0.99903 & \\
\hline Reletwe Denaing = & 1.0556 & 1.0556 & \\
\hline Gras FV (DRY) - & 927 & $\boldsymbol{2 9}$ & \\
\hline$G \operatorname{HV}($ SAT. $)=$ & 91.1 & 913 & \\
\hline Wobse lodax = & 9.8 & 90.0 & \\
\hline Nat HV $(D y)=$ & 29.8 & 90.0 & \\
\hline $\operatorname{Na} \operatorname{EV}(\operatorname{Ser})=$ & 883 & *8s & \\
\hline \multicolumn{4}{|c|}{ Nowe: All blet values we beton } \\
\hline \multicolumn{4}{|c|}{ ND. No Decond } \\
\hline \multicolumn{4}{|c|}{ 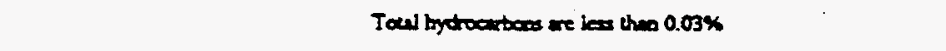 } \\
\hline
\end{tabular}




\begin{tabular}{|c|c|c|c|}
\hline & 3 Anatysis & Ges Curoena & anphy \\
\hline $\begin{array}{l}\text { IGT Sen } \\
\text { Samph } \\
\text { D }\end{array}$ & Piat & $\begin{array}{l}10 / 17 / 96 \\
\text { Anilyst da }\end{array}$ & 08.00 \\
\hline Coaponent & $\operatorname{Man} \%$ & Dex $\ln x$ & Worgte $\%$ \\
\hline Bdinen & N & $0.001 \%$ & ס \\
\hline Aydraper & 100\% & $0.0 \times 3$ & $146 \%$ \\
\hline 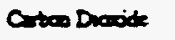 & $\Delta 4 x$ & $0.003 x$ & cs9s \\
\hline $8=$ & & $0.003 x$ & \\
\hline Ether & & $0.03 x$ & \\
\hline$a_{x p o d a r s e}$ & $172 \%$ & $0.03 x$ & 19.046 \\
\hline 150 & TدS* & $0.03 x$ & $705 \%$ \\
\hline Natex & & $0.08 x$ & \\
\hline C.roo Mosocoide & $24 \%$ & $0.03 x$ & $406 \%$ \\
\hline Etyox & & $0.002 x$ & \\
\hline Propere & & $0.002 \times$ & \\
\hline Propen: & & $0.002 x$ & \\
\hline Proptore & & $0.002 x$ & \\
\hline Proper & & $0.002 \times$ & \\
\hline ibverex & & $0.000 \times 6$ & \\
\hline$-8 \times x=$ & & $0.002 x$ & \\
\hline 1-Benex & & $0.002 x$ & \\
\hline ibecose & & $0.0002 x$ & \\
\hline Tr=-2plexe & & $0.005 x$ & \\
\hline C-2beax & & $0.002 \times$ & \\
\hline 13-Bundiex & & $0.0003 x$ & \\
\hline$=0$ & & $0.001 \%$ & \\
\hline Ptowine & & $0.000 x$ & \\
\hline 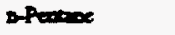 & & $0.002 x$ & \\
\hline Penowe: & & $0.002 \times$ & \\
\hline Rode Phe & & $0.000 x$ & \\
\hline Hydrem Sulfids & c.sonse & $0.0001 x$ & $0.0004 \%$ \\
\hline Catrogs surate & t.encose & $0.0001 x$ & .0136 \\
\hline Underifod & & $0.001 x$ & \\
\hline Wea & D & $0.001 \%$ & م \\
\hline
\end{tabular}

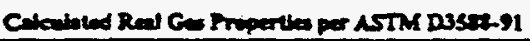

Temp (T) 6.0

Pren (pin) $140 \mathrm{ar} 1<7$

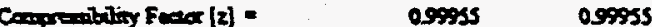

Reloive Dasidy = $\quad 1.0033$

Gra FV (DRY * $\quad 16.6 \quad 167$

Gon EV (SAI.) - $\quad 163 \quad 16.4$

Wobbe ladex = $\quad 16.6 \quad 16.6$

Ne HV (Dry) = $\quad 16.0 \quad 16.1$

Net $8 V(5 x)=\quad$ 15.8 $\quad 15.8$

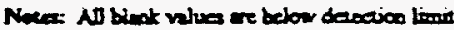

ND. Nox Determined

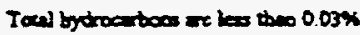




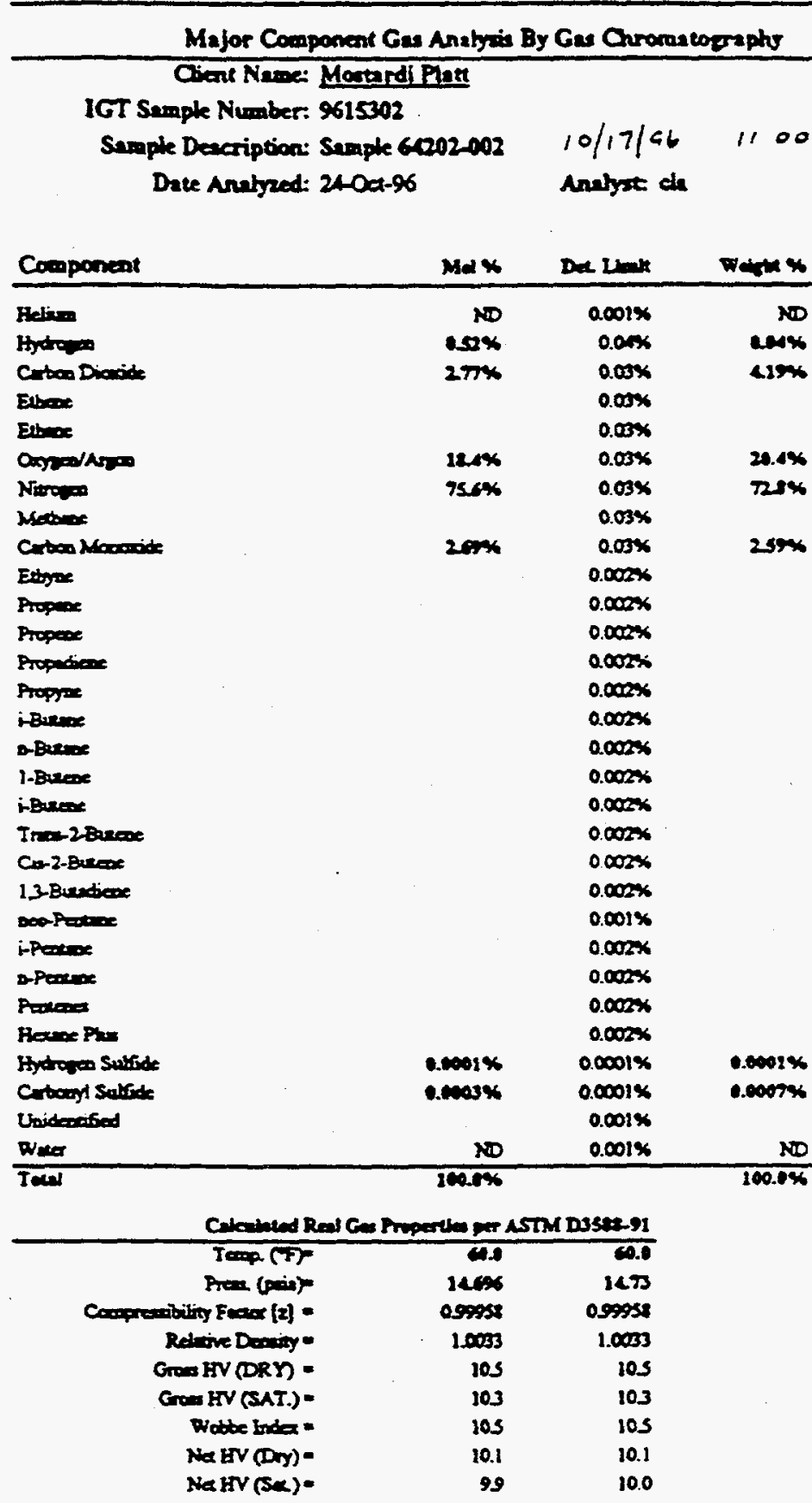

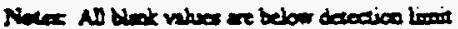

ND. Na Determined

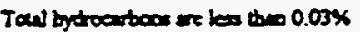




\begin{tabular}{|c|c|c|}
\hline & Gas Crromito & graphy \\
\hline $\begin{array}{r}\text { IGT Se } \\
\text { Sempk } \\
\text { D }\end{array}$ & $\begin{array}{l}10 / 17 / 96 \\
\text { Anabed da }\end{array}$ & 1300 \\
\hline Component & Dec Lant & Wrature $\%$ \\
\hline 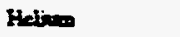 & $0.001 x$ & ס \\
\hline Fydrowe & $0.00 \times x$ & $031 \%$ \\
\hline Crboo Dincite & $0.03 x$ & $357 \%$ \\
\hline Entere & $0.00 \%$ & \\
\hline Ethere & $0.000 x$ & \\
\hline$O_{x y}=D_{1} x_{2}=$ & $0.03 x$ & 1.536 \\
\hline Norose & $0.03 x$ & $42 x$ \\
\hline Methex & $0.03 \times$ & \\
\hline C.t+o Mosodite & $0.03 x$ & 2324 \\
\hline Ethyse & $0.002 x$ & \\
\hline Proper & $0.002 \%$ & \\
\hline Propere & $0.002 x$ & \\
\hline Propudiene & $0.002 x$ & \\
\hline Proprex & $0.002 x$ & \\
\hline$j-\operatorname{Ban} x$ & $0.002 x$ & \\
\hline D-Bune & $0.005 x$ & \\
\hline 1-Bureax & $0.000 x$ & \\
\hline i-Bvecex & $0.005 x$ & \\
\hline Inte-2Beerese & $0.005 x$ & \\
\hline Cit-2-Buene & $0002 x$ & \\
\hline 13-Bundiant & $0.002 x$ & \\
\hline 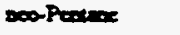 & $0.001 \%$ & \\
\hline iptentox & $0.002 x$ & \\
\hline D-Prantex & $0.002 x$ & \\
\hline Peorong & $0.002 x$ & \\
\hline Hexine Phe & $0.000 x$ & \\
\hline Hydroper Sutsete & $0.0001 \times$ & $0.0016 \%$ \\
\hline Cutconyl Subsict & $0.0001 \%$ & 0.00694 \\
\hline Ubidenofied & $0.001 \%$ & \\
\hline Wmer & $0.001 \%$ & No \\
\hline
\end{tabular}

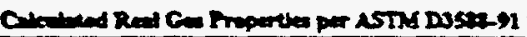

\begin{tabular}{|c|c|c|}
\hline $\operatorname{Tag}(T)$ & 60 & की \\
\hline Prex (pin) & I4ex & $1<7$ \\
\hline 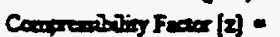 & 09909 & osses \\
\hline Reloinc Do. & 1.0556 & 1.0656 \\
\hline $\operatorname{cres} \sin (\operatorname{Dr})=$ & 99.1 & \\
\hline Gra EV (SAT.) $=$ & 973 & 97 \\
\hline Wobte bdex = & 96.0 & 96 \\
\hline NaEV $(D y)=$ & 95.8 & 96. \\
\hline Net $\operatorname{RV}(\mathrm{SC})=$ & 94.1 & 94 \\
\hline
\end{tabular}

Nolex All biate vives tre beloor decocion limit ND. Nox Dearoined

Teeal bydroctom are les the $0.03 \%$ 


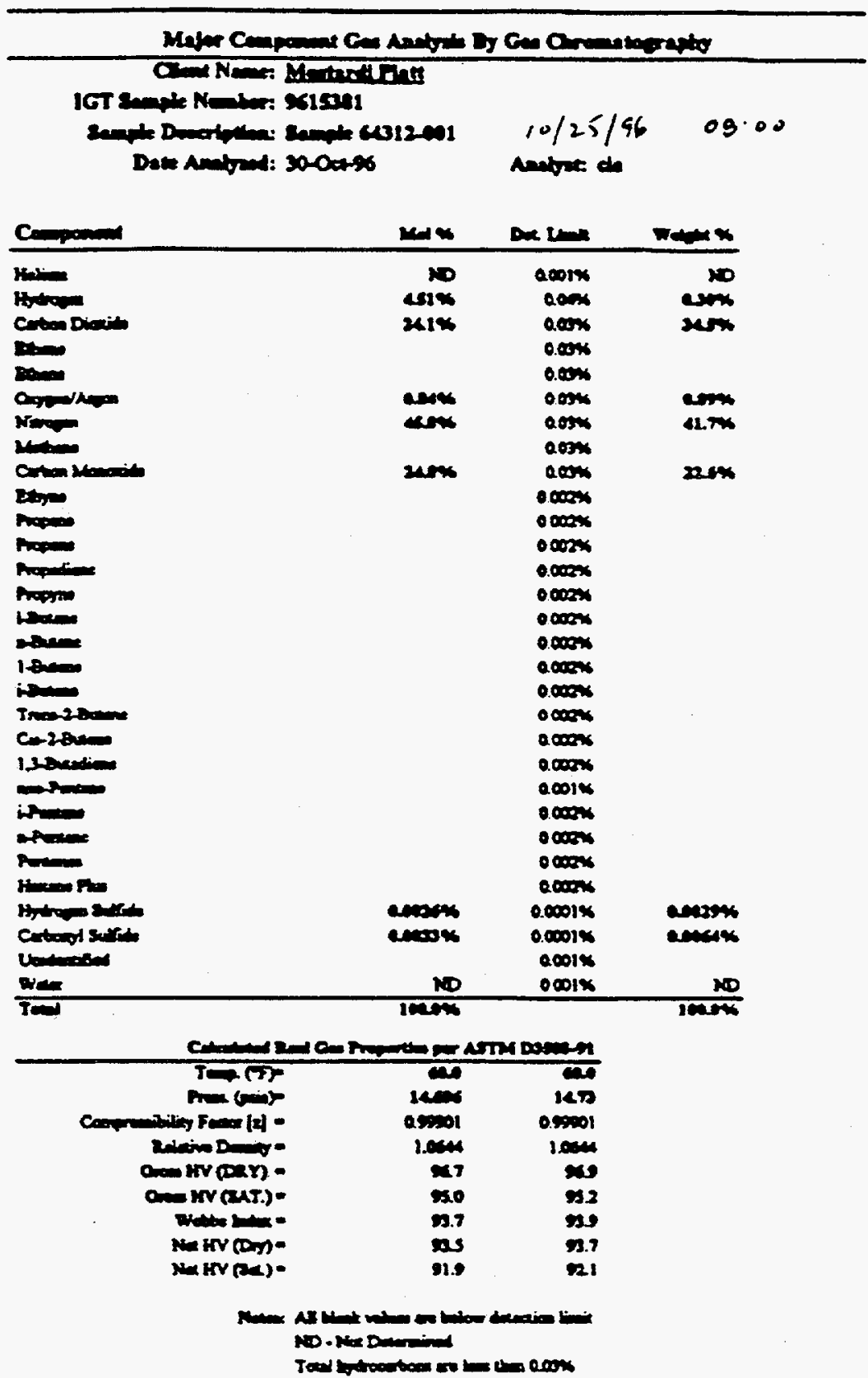




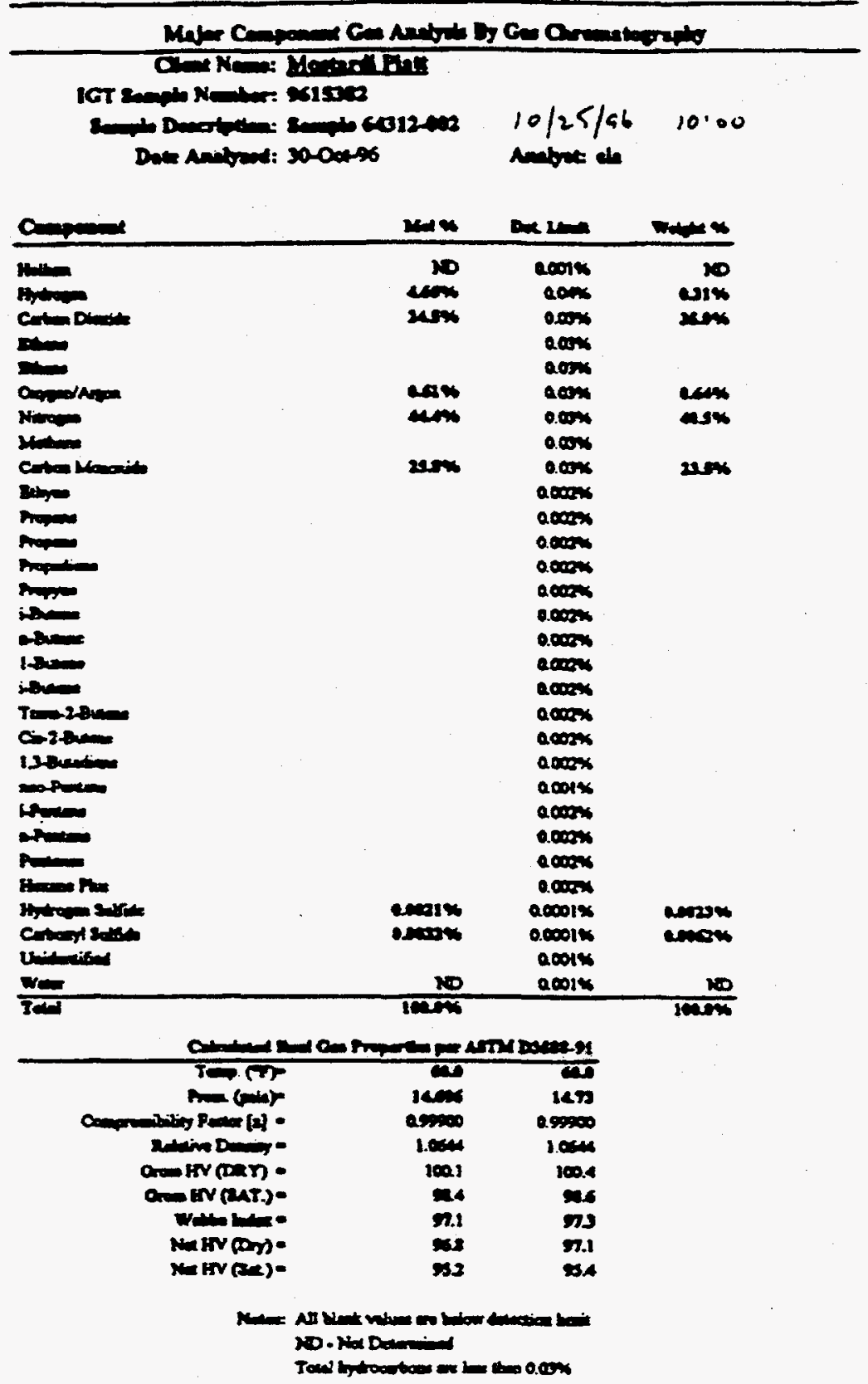




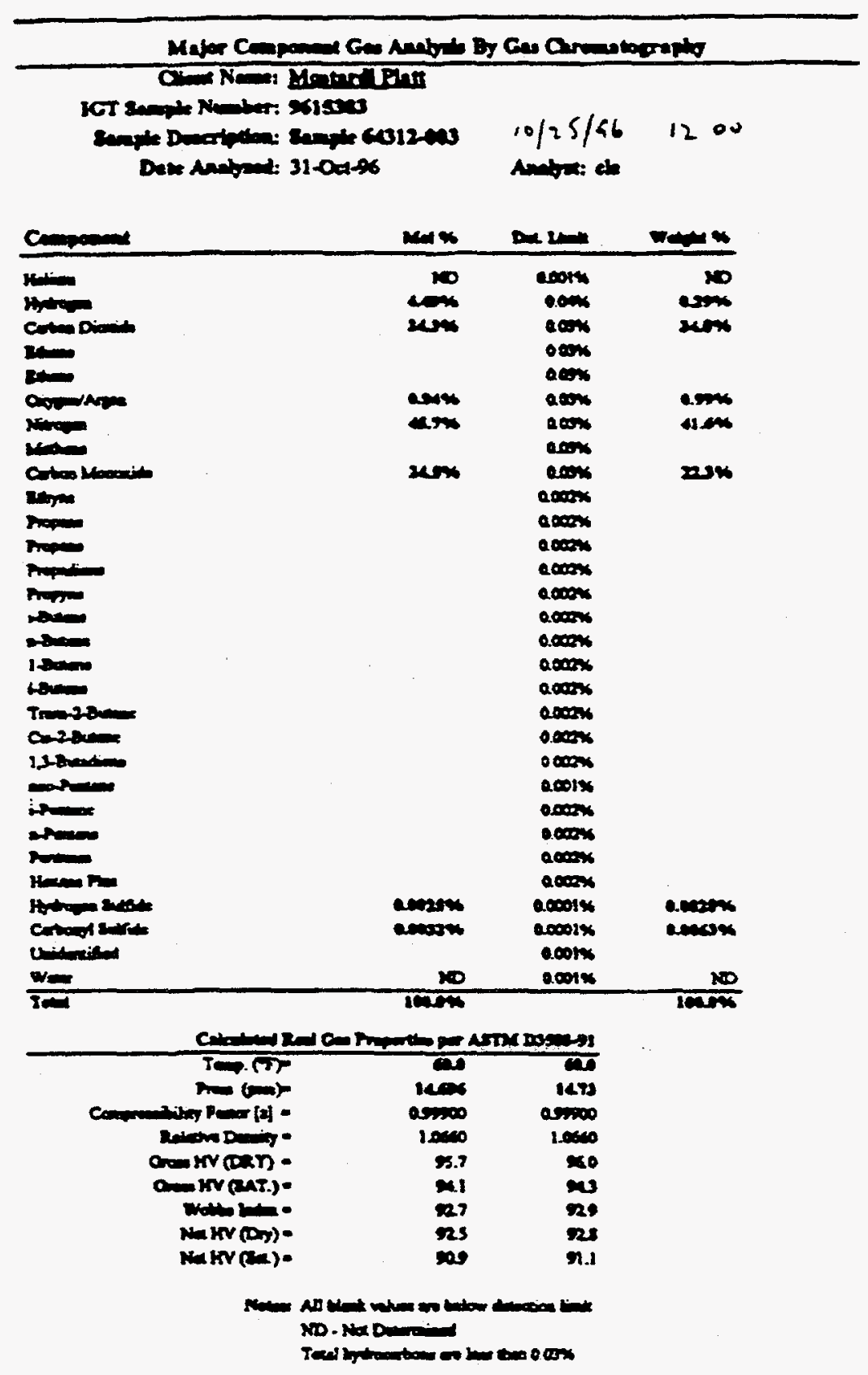




\title{
TRACE SULFUR DETERMINATION BY GAS CHROMATOGRAPHY
}

\author{
Client Name: Mostandi Platt
}

IGT Sample Number: 9614961

Sample Description: Sample 64017-001

Date Analyzed: 7-0et-96

Analyst: cla

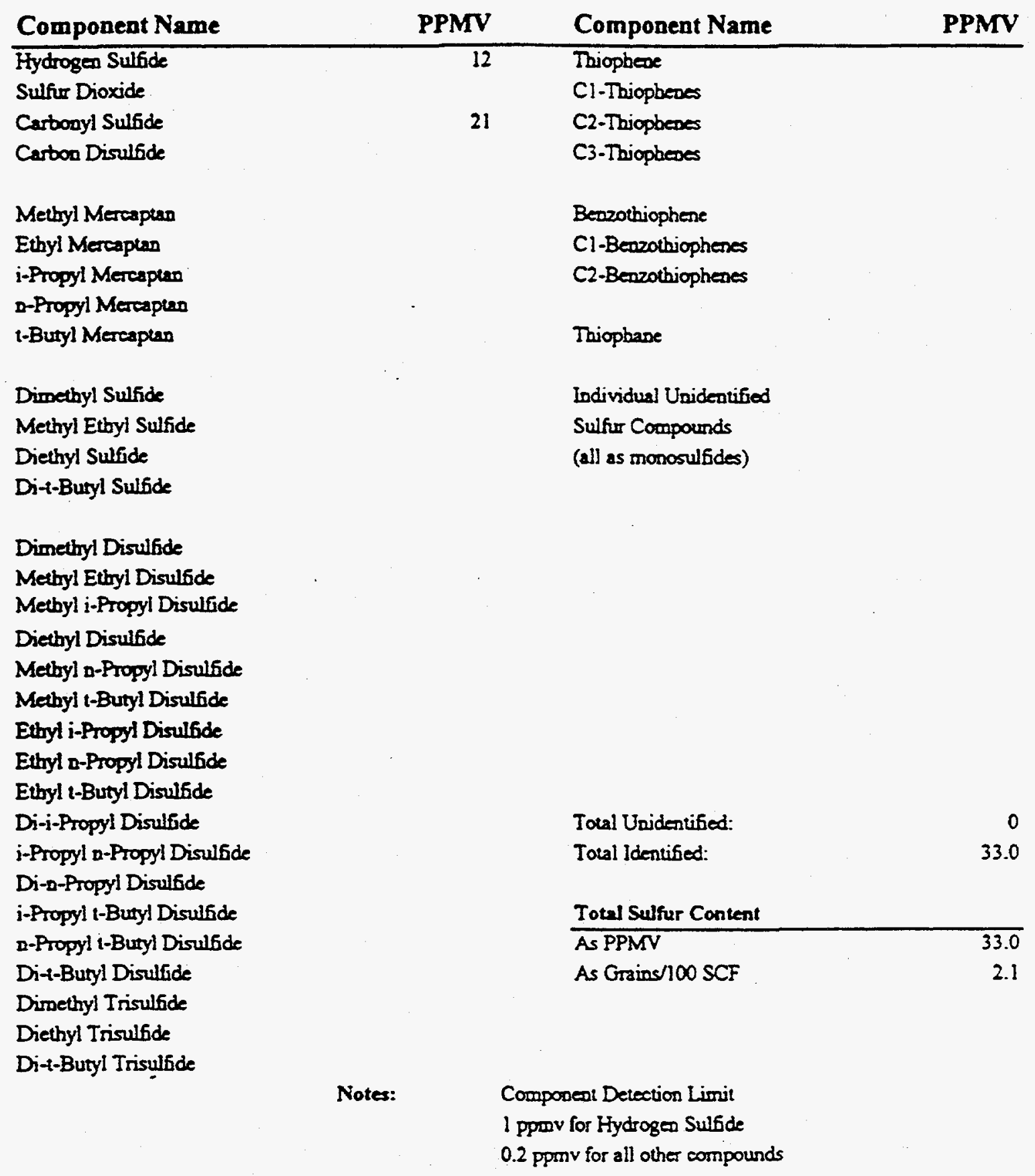




\title{
TRACE SULFUR DETERMINATION BY GAS CHROMATOGRAPHY
}

\author{
Client Name: Mostardi Platt \\ IGT Sample Number: 9614962 \\ Sample Description: Sample 64017-002 10/3/46 1/.0 \\ Date Analyzed: 7-0et-96 \\ Analyst: cia
}

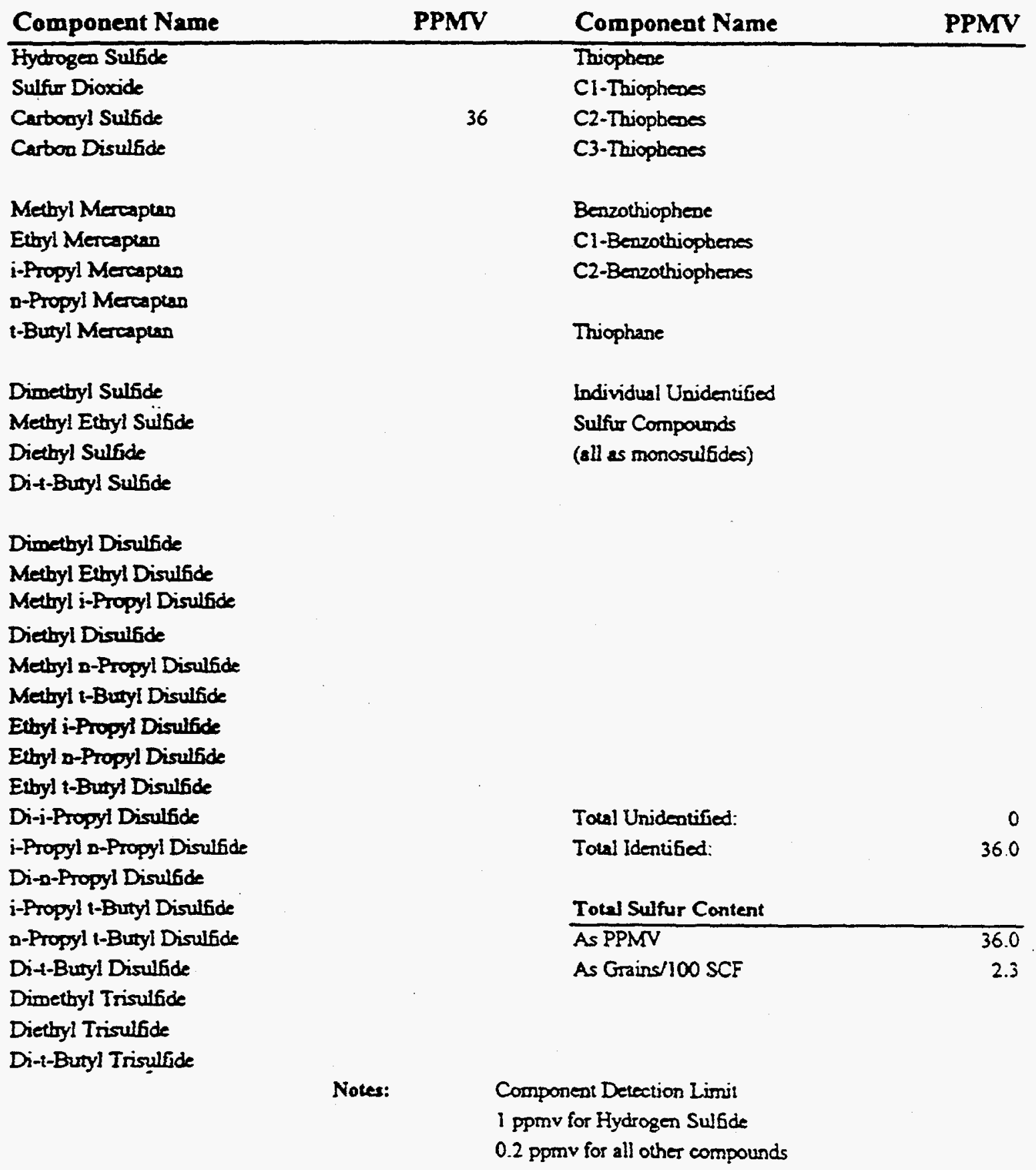


TRACE SULFUR DETERMINATION BY GAS CHROMATOGRAPHY

\author{
Client Name: Mostandi Plant \\ IGT Sample Number: 9614963 \\ Sample Description: Sample 64017-003 10/3/46 /3:00 \\ Date Analyzed: 7-0ct-96 \\ Analyst: cla
}

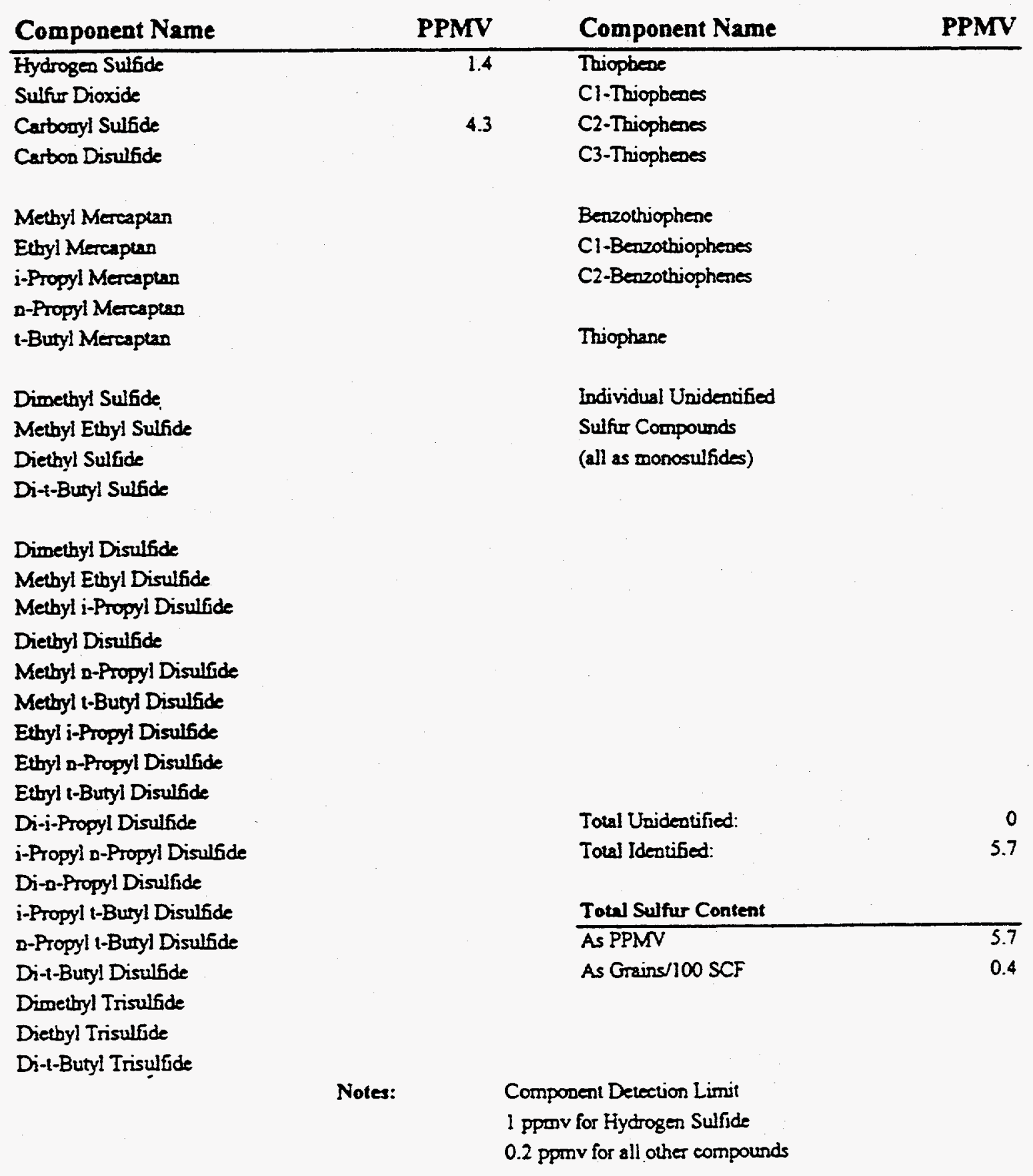




\section{TRACE SULFUR DETERMINATION BY GAS CHROMATOGRAPHY}

Client Name: Mostardi Plat

IGT Sample Number: 9615121

Sample Description: Sample 64113-001

Date Analyzed: 15-0ct.96

\section{Analyst: cla}

$$
10 / 9 / 96 \quad 03: 00
$$

\section{Component Name}

Hydrogen Sulfide

Sulfur Dioxide

Cartonyl Sulfide

Carbon Disulfide

Methyl Meraplan

Etbyl Mercaptan

i-Propyl Mercaptan

D-Propyl Mercaptan

t-Butyl Mercaptan

Dimethyl Sulfide

Methyl Ethyl Sulfide

Diethyl Sulfide

Dit-Butyl Sulfide

Dimethyl Disulfide

Methyl Ethyl Disulfide

Methyl i-Propyl Disulfide

Diettryi Disulfide

Methyl a-Propyl Disulfide

Methyl t-Buryl Disulfide

Ettryl i-Propyl Disulfide

Ethyl a-Propyl Disulfide

Ethyl t-Butyl Disulfide

Di-i-Propyl Disulfide

i-Propyl n-Propyl Disulfide

Di-n-Propyl Disulfide

i-Propyl t-Butyl Disulfide

-Propyl :-Butyl Disulfide

Di-t-Buryl Disulfide

Dimetbyl Trisulfide

Diechyl Trisulfide

Di-i-Butyl Trisulfide
PPMV

Component Name

PPMV
Thiopbece
Cl-Thiophenes
C2-Thiophenes
C3-Taiophenes

Benzothiopbene

C1-Benzotbiophenes

C2-Benzothiophenes

Thiopbane

Individual Unidentifed

Sulfur Compousds

(all as monosulfides)
Toual Unidentified: $\quad 0$

Total Identified. $\quad 34.0$

Total Sulfur Cootent

As PPMV 34.0

As Grains 100 SCF

2.1

Notes: Component Detection Limit

I pponv for Hydrogen Sulfide

0.2 pponv for all ocher compounds 
$1 G T$ Institute of Gas Technology Analytical Report

\section{TRACE SULFUR DETERMINATION BY GAS CHROMATOGRAPHY}

Client Name: Mostardi Plan

IGT Sample Number: 9615122

Sample Description: Sample 64113-002 10/9/.6 11:00

Date Analyzed: 15-0a-96

Analyst: cla

\section{Component Name}

Hydrogen Sulfide

Sulfur Dioxide

Carbonyl Sulfide

Carbon Disulfide

Metbyl Mercaptan

Etby! Mereaptan

i-Propyl Mercaptan

D-Propyl Mercaptao

t-Buryl Mercapuan

Dimethyl Sulfide

Methyl Etbyl Sulfide

Diechyl Sulfide

Dit-Butyl Sulfide

Dimethyl Disulede

Methyl Ethyl Disulifie

Methyl i-Propyl Disulfide

Diethyl Disulfide

Methyl o-Propyl Disulfide

Mechyl t-Butyl Disulfide

Ethyl i-Propyl Disulfide

Etryl a-Propyl Disulfide

Etbyl t-Butyl Disulfide

Di-i-Propyl Disulfide

i-Propyl n-Propyl Disulfide

Di-a-Propyl Disulfide

i-Propyl t-Butyl Disulfide

a-Propyl t-Butyl Disuifide

Di-t-Butyl Disulfide

Dimethyl Trisulfide

Diethyl Trisulfide

Di-t-Butyl Trisulfide
PPMV

Component Name

PPMV

Thiophere

C1-Thiophenes

4.3 C2-Thiophenes

C3-Thiophenes

Beczothiophene

Cl-Benzochiopbenes

C2-Benzochiophenes

Thioptane

Individual Uaidentifed

Sulfur Compourds

(all as monosulfides)
Total Unidentified:

Total ldeatified:

Total Salfur Content

\begin{tabular}{ll}
\hline As PPMV & 5.4 \\
As Grains $/ 100$ SCF & 0.3
\end{tabular}

Notes: Component Detection Limit

1 pprnv for Hydrogen Sulfide

0.2 pperv for all ocher compounds 


\section{TRACE SULFUR DETERMINATION BY GAS CHROMATOGRAPHY}

Client Name: Mostardi Plant

IGT Sample Number: 9615123

Sample Description: Sample 64113-003 /0/4/46 13:00

Date Analyzed: 15-Oct-96

Analyst: ela

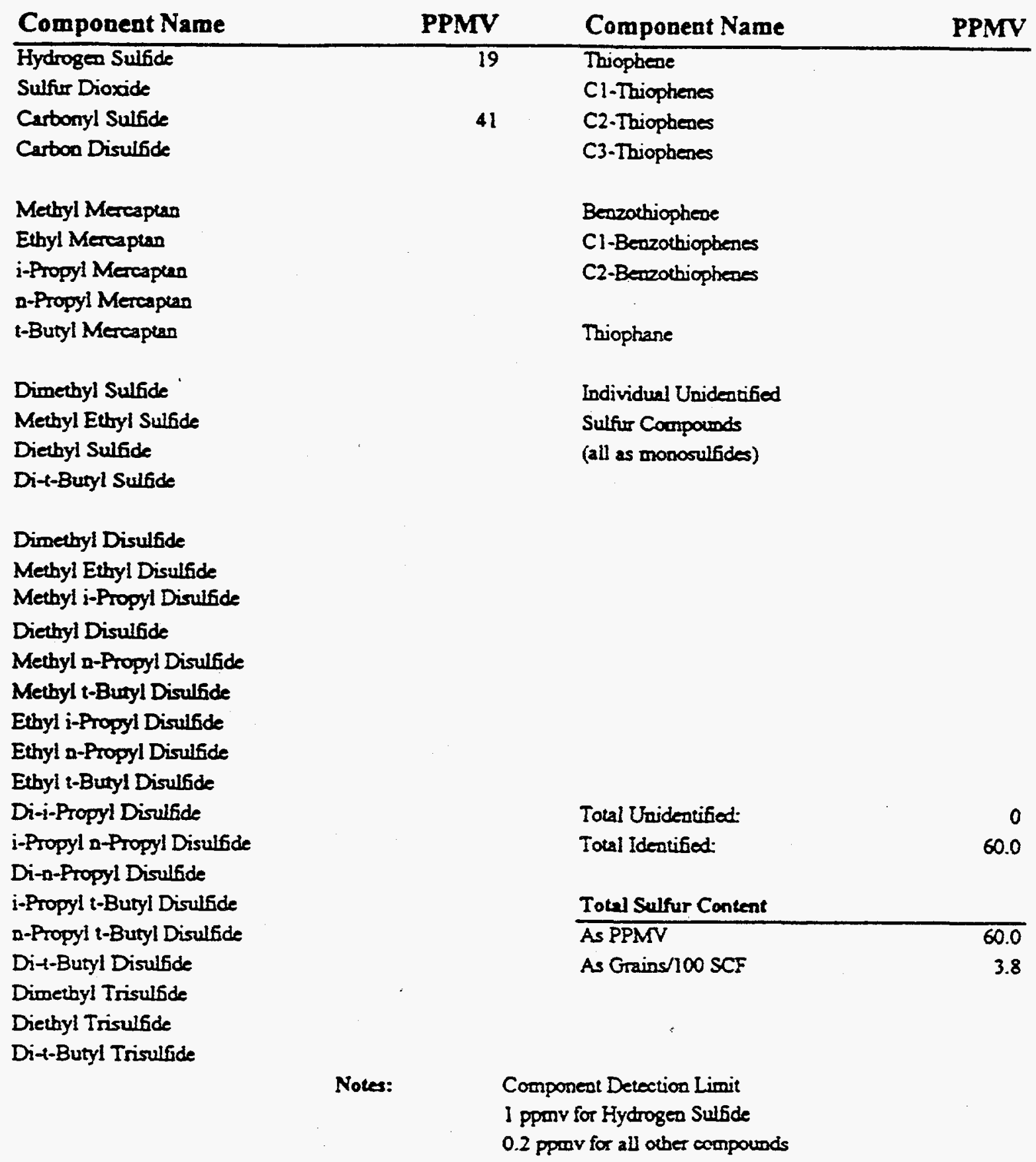




\title{
TRACE SULFUR DETERMINATION BY GAS CHROMATOGRAPHY
}

\author{
Client Name: Mostardi Plan
}

IGT Sample Number: 9615301

Sample Description: Sample 64202-001 1/17/96 08:00

Date Analyzed: 23-0c-96

Analyst: da

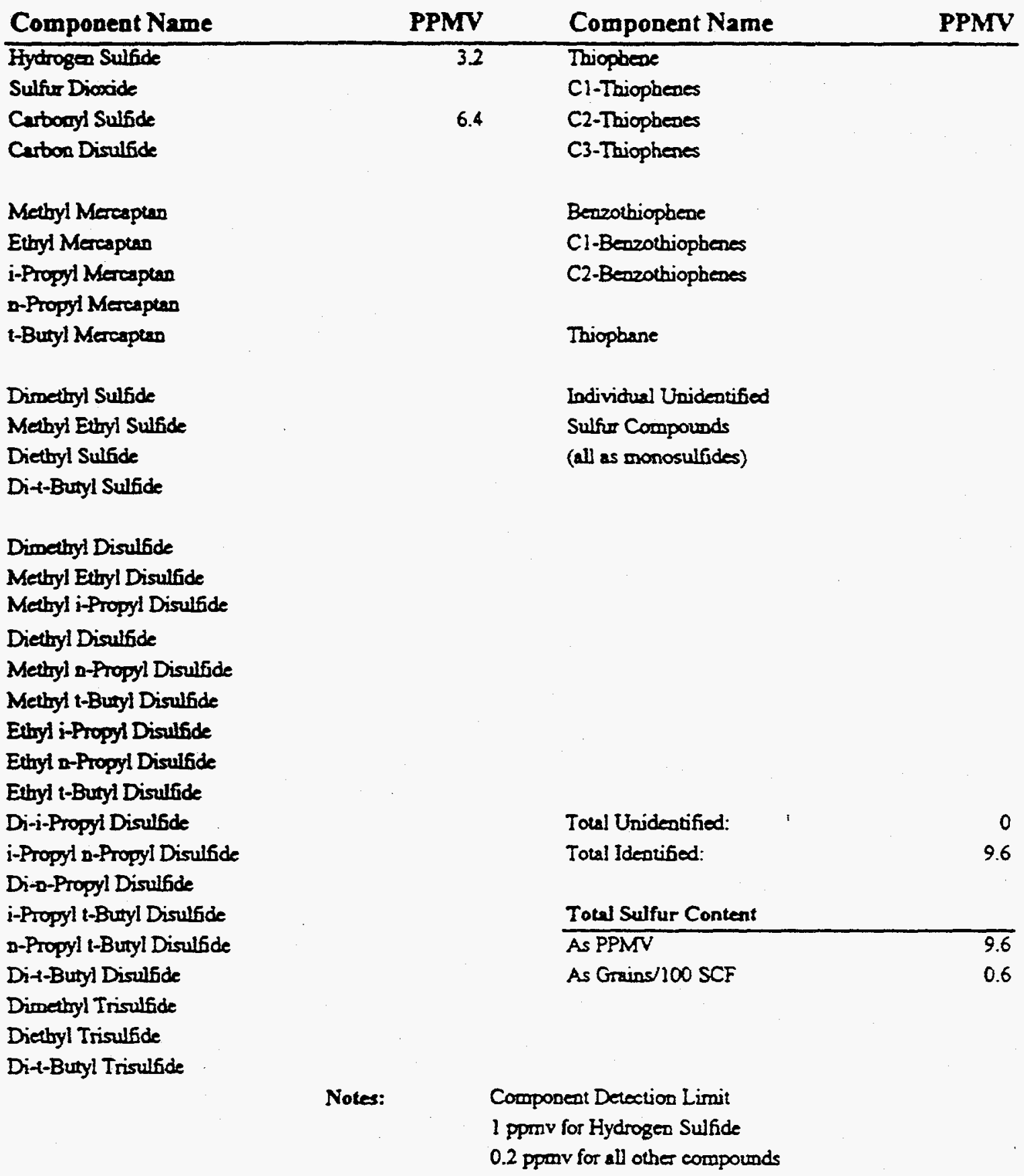




\section{TRACE SULFUR DETERMINATION BY GAS CHROMATOGRAPFY}

Client Name: Mostardi Plat

IGT Sample Number, 9615302

Sample Description: Sample 64202-002 10/17/96 11:00

Date Analyzed: 23-Oct-96

Analyst: cls

\begin{tabular}{|c|c|c|c|}
\hline Component Name & PPMV & Component Name & PPMV \\
\hline Hydrogen Sulfide & 1 & Thiophede & \\
\hline Sulfur Dioxide & & C1-Thiophenes & \\
\hline Carbonyl Sulfide & 3.3 & C2-Thiophedes & \\
\hline Carbon Disulfide & & C3-Thiophenes & \\
\hline Metbyl Mercaptan & & Benzothiophear & \\
\hline Ethyl Mercaptas & & C1-Benzothiophenes & \\
\hline i-Propyl Mercapesn & & C2-Benzothiophenes & \\
\hline \multicolumn{4}{|l|}{ D-Propyl Merapien } \\
\hline t-Butyl Mereaptas & & Thiophane & \\
\hline Dimetbyl Sulfide & & Individual Unidentifod & \\
\hline Metbyl Ethyl Sulfide & & Sulfur Compounds & \\
\hline Diethyl Sulfide & & (all as monosulfides) & \\
\hline \multicolumn{4}{|l|}{ Di--Buryl Sulfide } \\
\hline \multicolumn{4}{|l|}{ Dimethyl Disulfide } \\
\hline \multicolumn{4}{|l|}{$\begin{array}{l}\text { Methyl Etbyl Disulfide } \\
\text { Metbyl i-Propyl Disulfide }\end{array}$} \\
\hline \multicolumn{4}{|l|}{ Dietbyl Disulfide } \\
\hline \multicolumn{4}{|l|}{ Methyl a-Propyl Disulade } \\
\hline \multicolumn{4}{|l|}{ Metbyl i-Butyl Disulfide } \\
\hline \multicolumn{4}{|l|}{ Ethyl i-Propyl Disulfide } \\
\hline \multicolumn{4}{|l|}{ Ettyl D-Propyl Disulfide } \\
\hline \multicolumn{4}{|l|}{ Ethyl i-Buryl Disulfde } \\
\hline Di-i-Propyl Disulfide & & Total Unidentified: & 0 \\
\hline i-Propyl o-Propyl Disulfide & & Total Identified: & 4.3 \\
\hline \multicolumn{4}{|l|}{ Di-n-Propyl Disulsde } \\
\hline i-Propyl i-Buryl Disulfide & & Total Sulfur Content & \\
\hline D-Propyl t-Butyl Disulfide & & As PPMV & 4.3 \\
\hline Di-t-Butyl Disulfide & & As Grains $1100 \mathrm{SCF}$ & 0.3 \\
\hline \multicolumn{4}{|l|}{ Dimethyl Trisulbide } \\
\hline \multicolumn{4}{|l|}{ Dietbyl Trisulade } \\
\hline \multicolumn{4}{|l|}{ Di-t-Butyl Trisulfide } \\
\hline \multirow{2}{*}{\multicolumn{4}{|c|}{$\begin{array}{l}\text { Component Detection Limit } \\
1 \text { ppenv for Hydrogan Sulfide }\end{array}$}} \\
\hline & & & \\
\hline & \multicolumn{3}{|c|}{$0.2 \mathrm{ppmv}$ for all other conpounds } \\
\hline
\end{tabular}




\title{
TRACE SULFUR DETERMINATION BY GAS CHROMATOGRAPHY
}

\author{
Client Name: Mostandi Plar \\ IGT Sample Number: 9615303 \\ Sample Description: Sample 64202-003 \\ $10 / 17 / 46 \quad 13: 00$ \\ Date Analyzed: 23-0at-96 \\ Analyst: cia
}

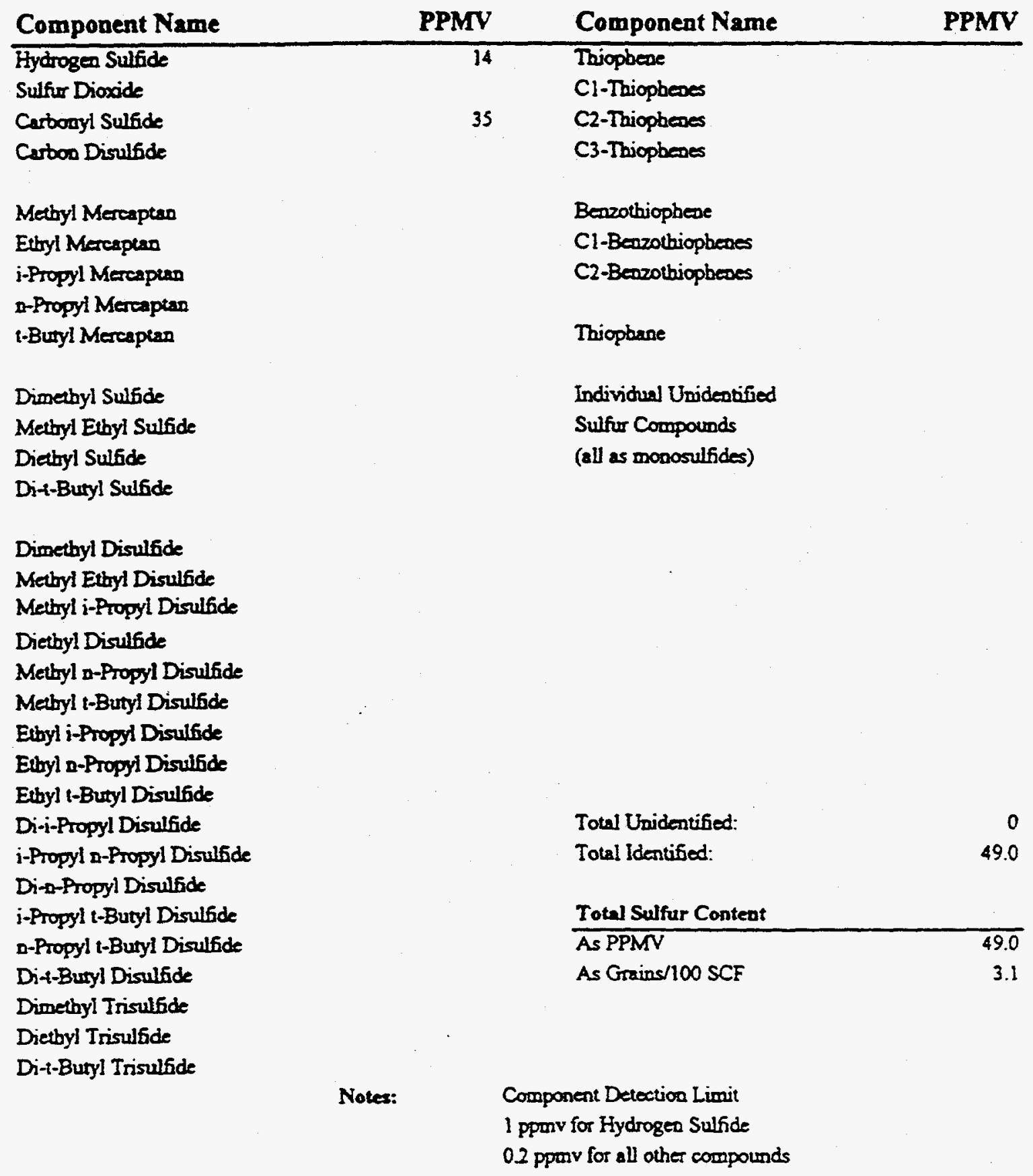




\title{
TRACL SULFUR DETERMINATION BY GAS CHROMATOGRAPHY
}

\author{
Client Name: Moundipin
}

IGT Sample Number: 9615381

Sample Description: Sample 64312001

Dale Andyred: 29-0a-96

Anabyat: de

$$
10 / 25 / 96 \quad 08: 00
$$

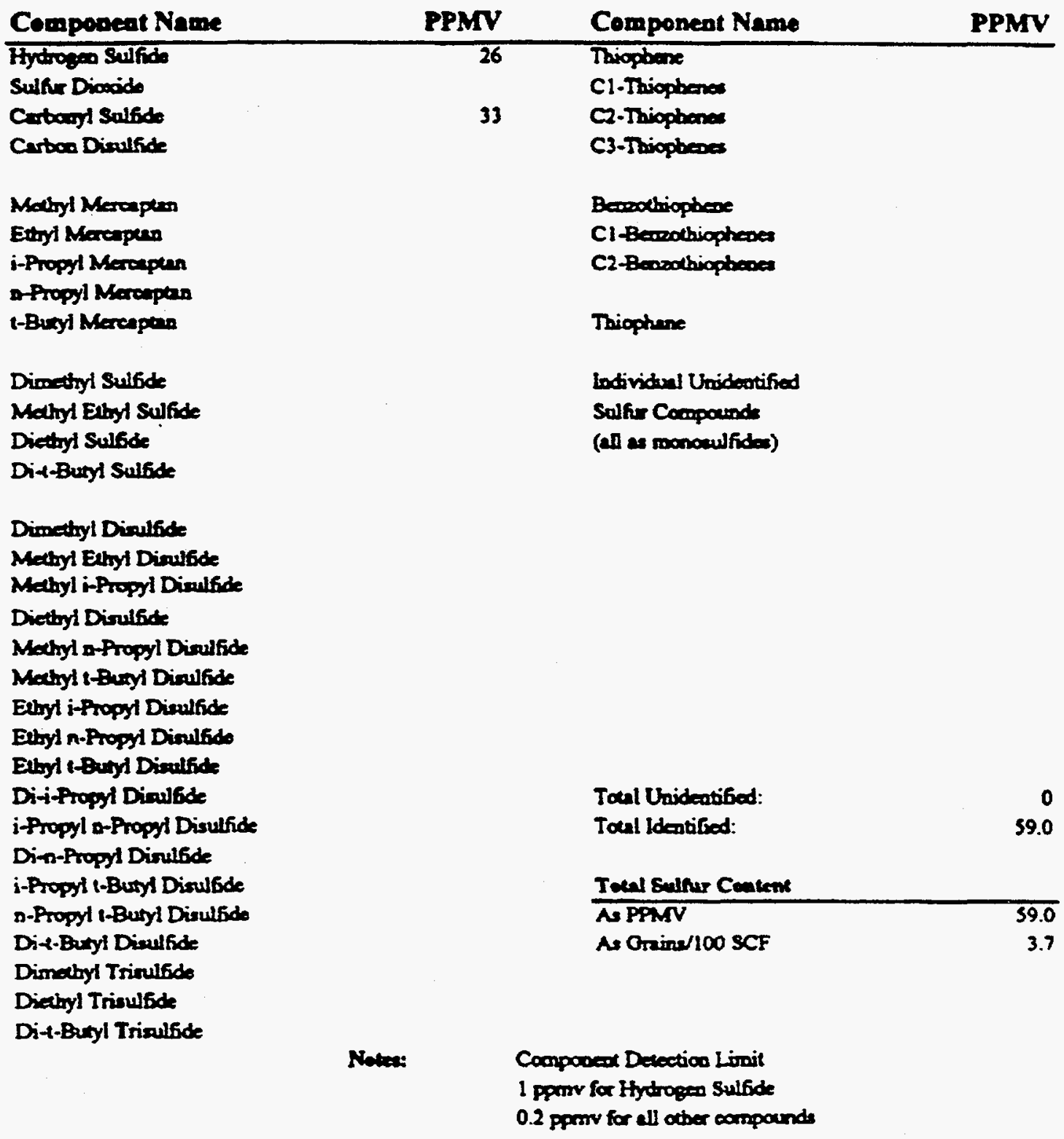




\section{TRACE SULFUR DETERMTATION BY GAS CIROMATOGRAPHY}

\section{Client Name: Mortondifun}

IGT Sample Number: $\$ 615382$

Sample Deseription: Semplo 64312-002

Date Analyzed: $3000-96$

Analyet: ch

$$
10 / 25 / 9610: 00
$$

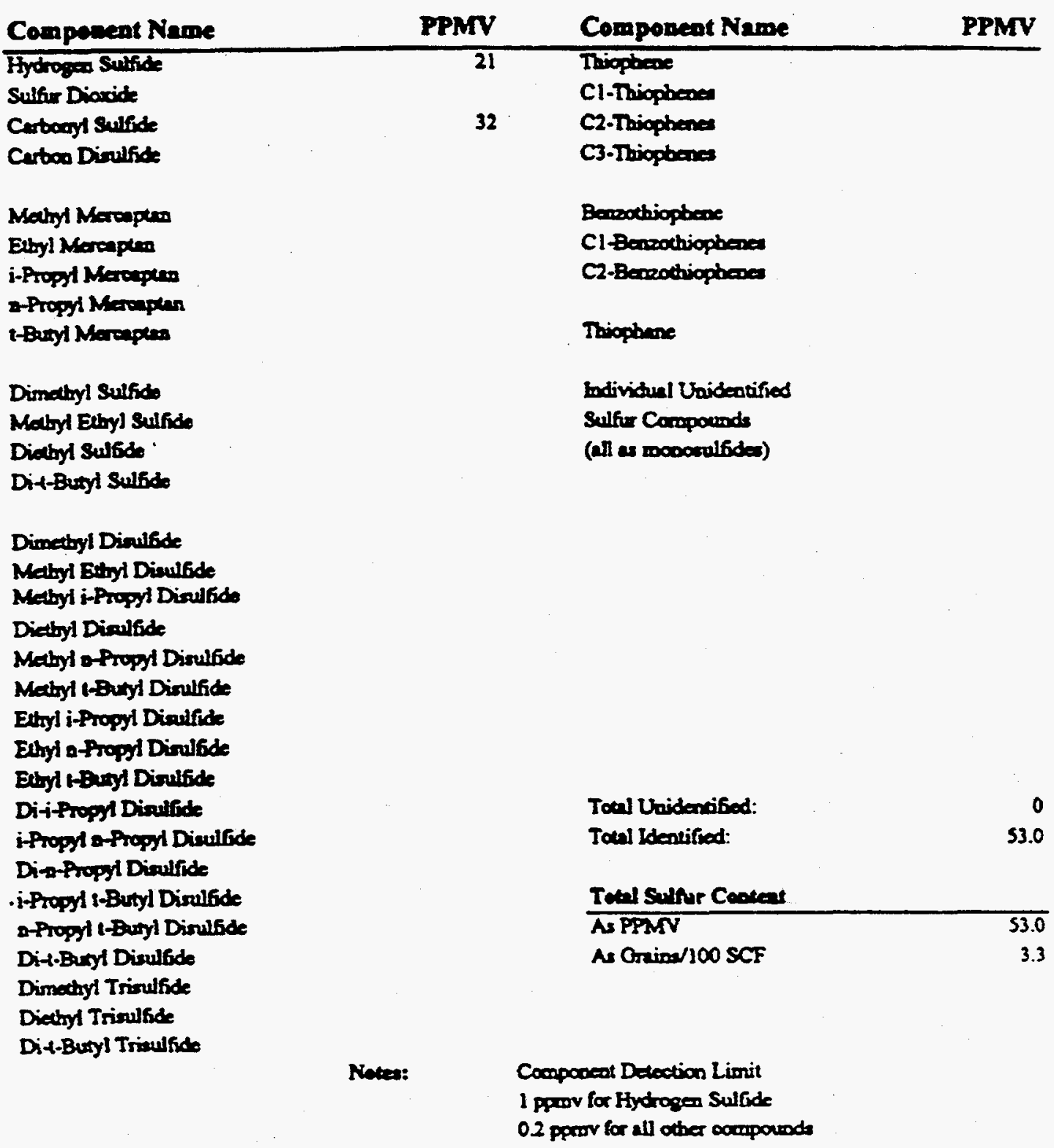


TRACE SULFUR DETERMINATION BY GAS CHROMATOGRAPHY

Client Name: Motuntiping

IGT Sample Number: $\$ 615383$

Sample Description: Semple 61312-003

Date Anahsed: $300 \times 0.96$

$10 / 25 / 46 \quad 12: 00$

Anclyst: do

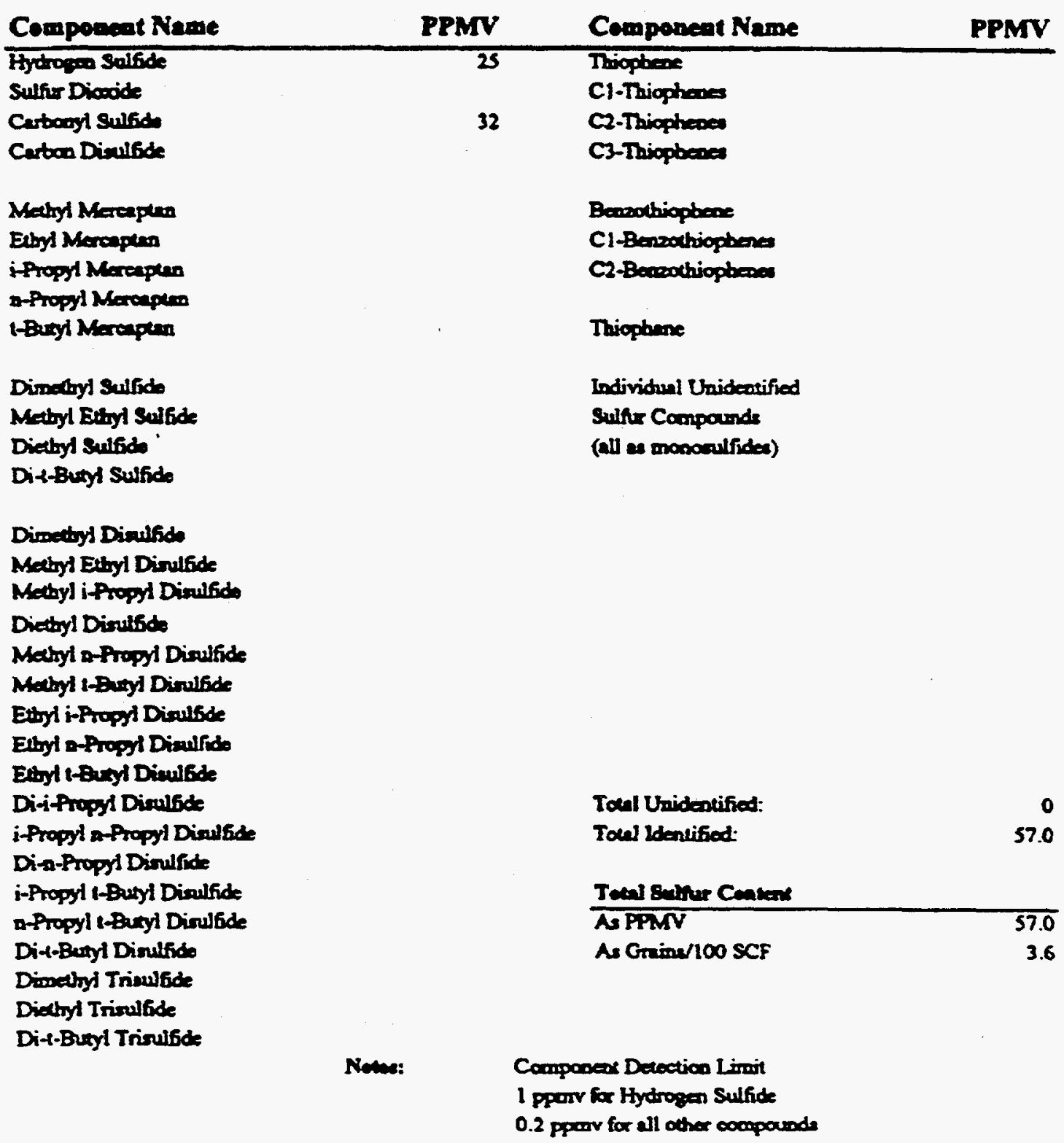


MOSTARDI-PLATT ASSOCIATES, INC.

Environmental Consultants

Read Instructions on Reverse Side Before Completing Form!

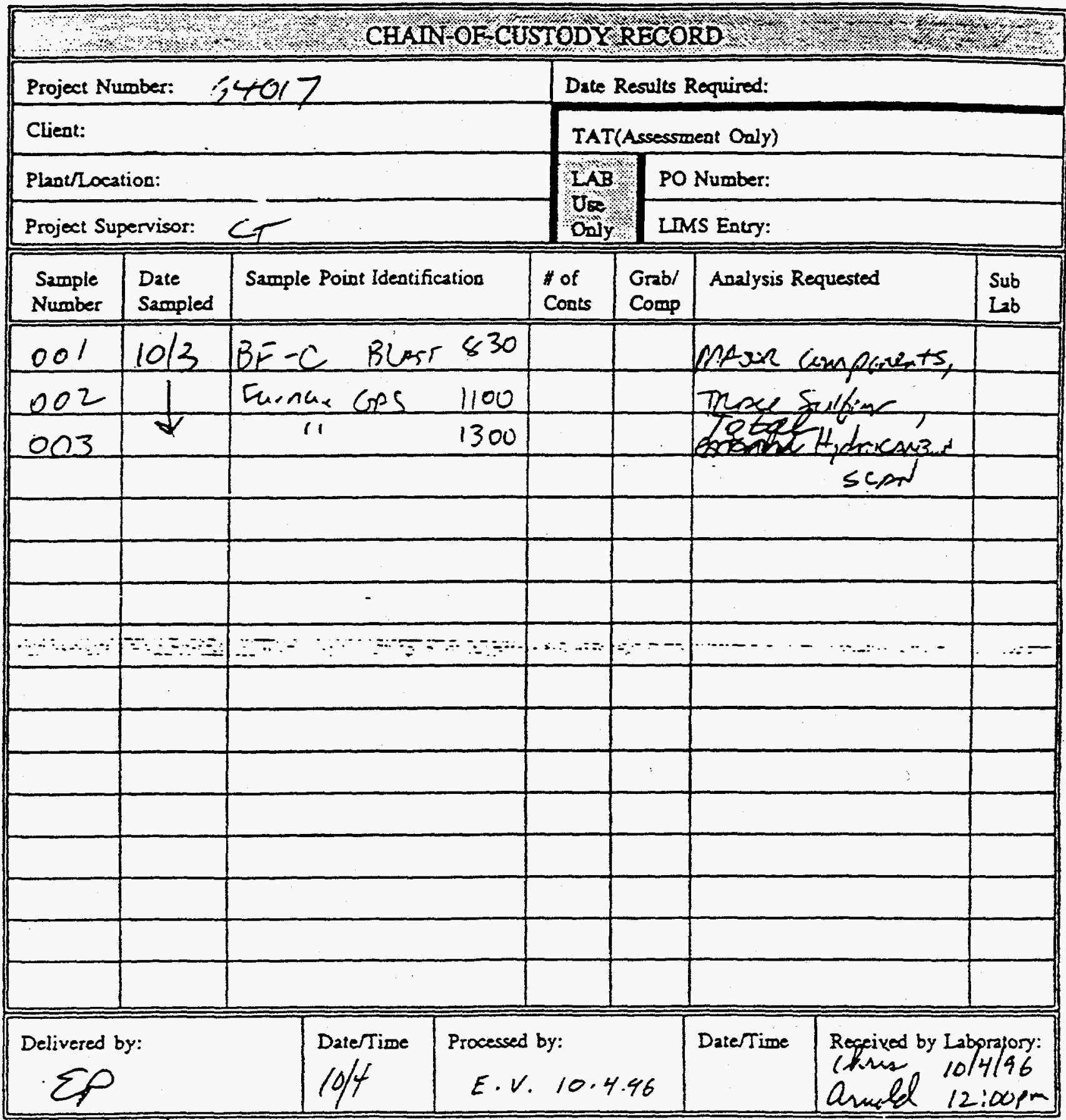

Special Instructions: 
MOSTARDI-PLATT ASSOCIATES, INC.

Environmental Consultants

$$
\angle 96-1512
$$

Read Instructions on Reverse Side Before Completing Form!

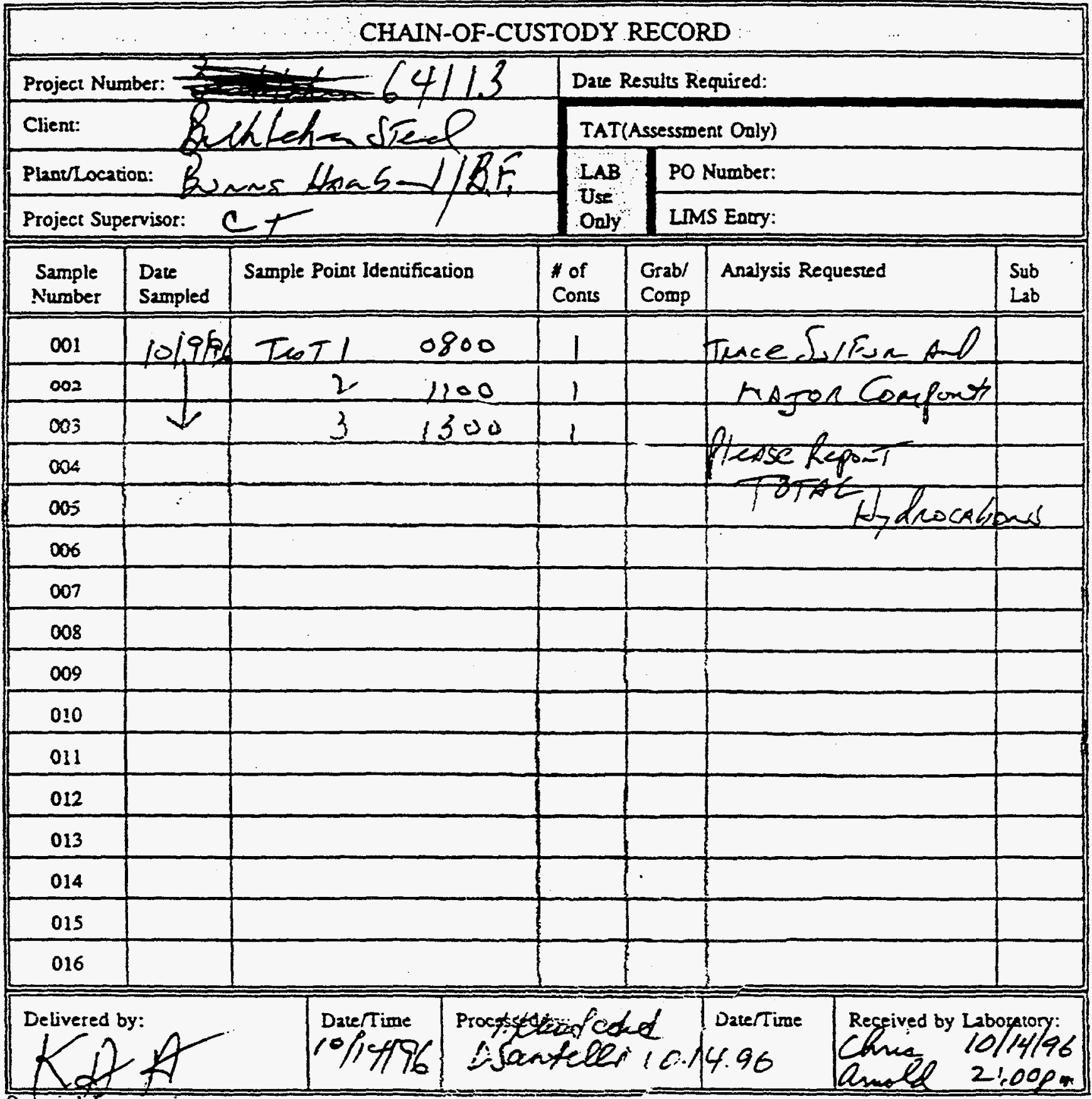

Special Instructions: 
MOSTARDI-PLATT ASSOCIATES, INC.

Environmental Consultants

$$
\angle 96-1530
$$

Read Instructions on Reverse Side Before Completing Form!

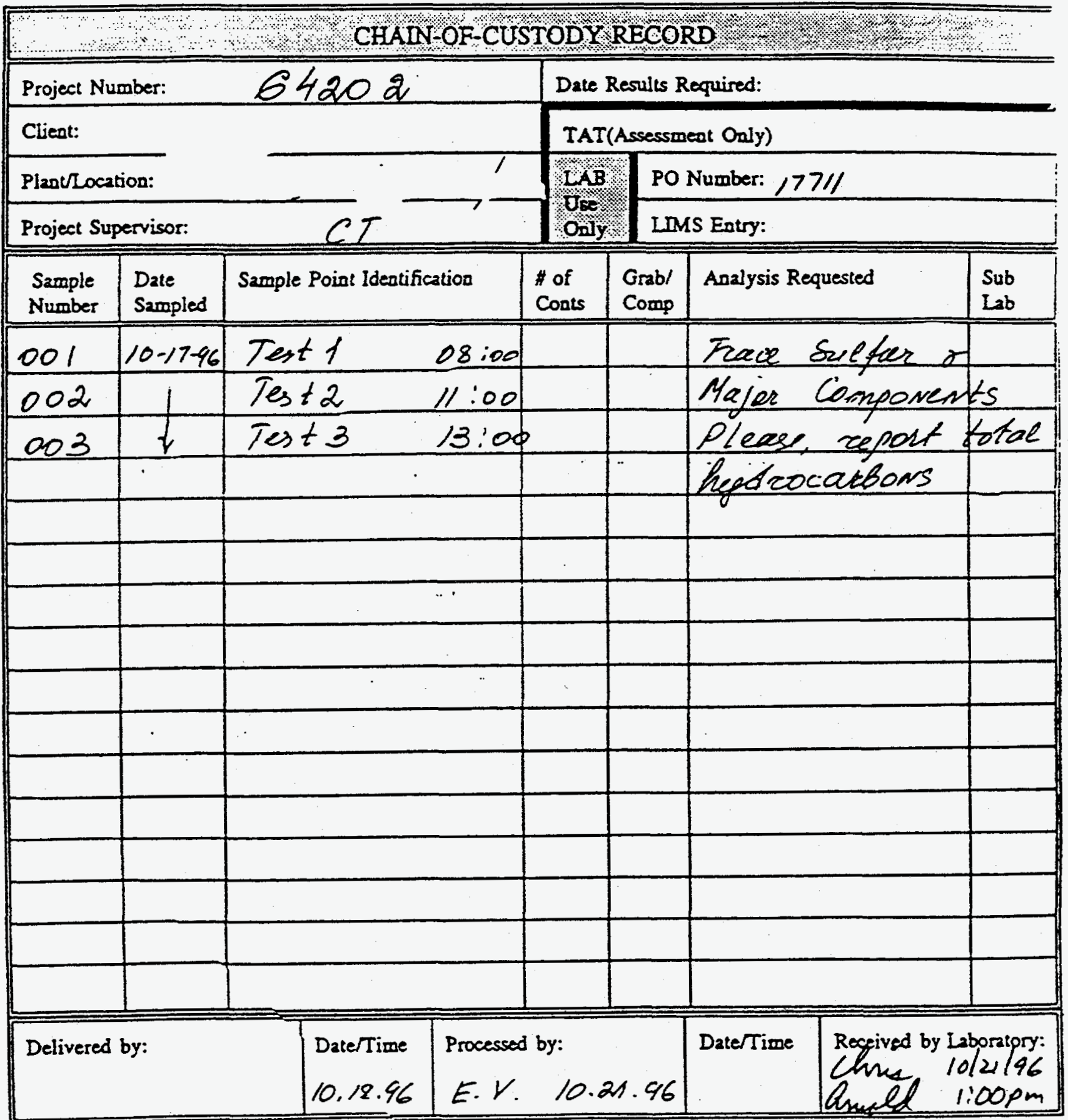

Special Instructions: 
MOSTARDI-PLATT ASSOCIATES, INC.

Environmental Consultants

4

Read Instructions on Reverse Side Before Completing Form!

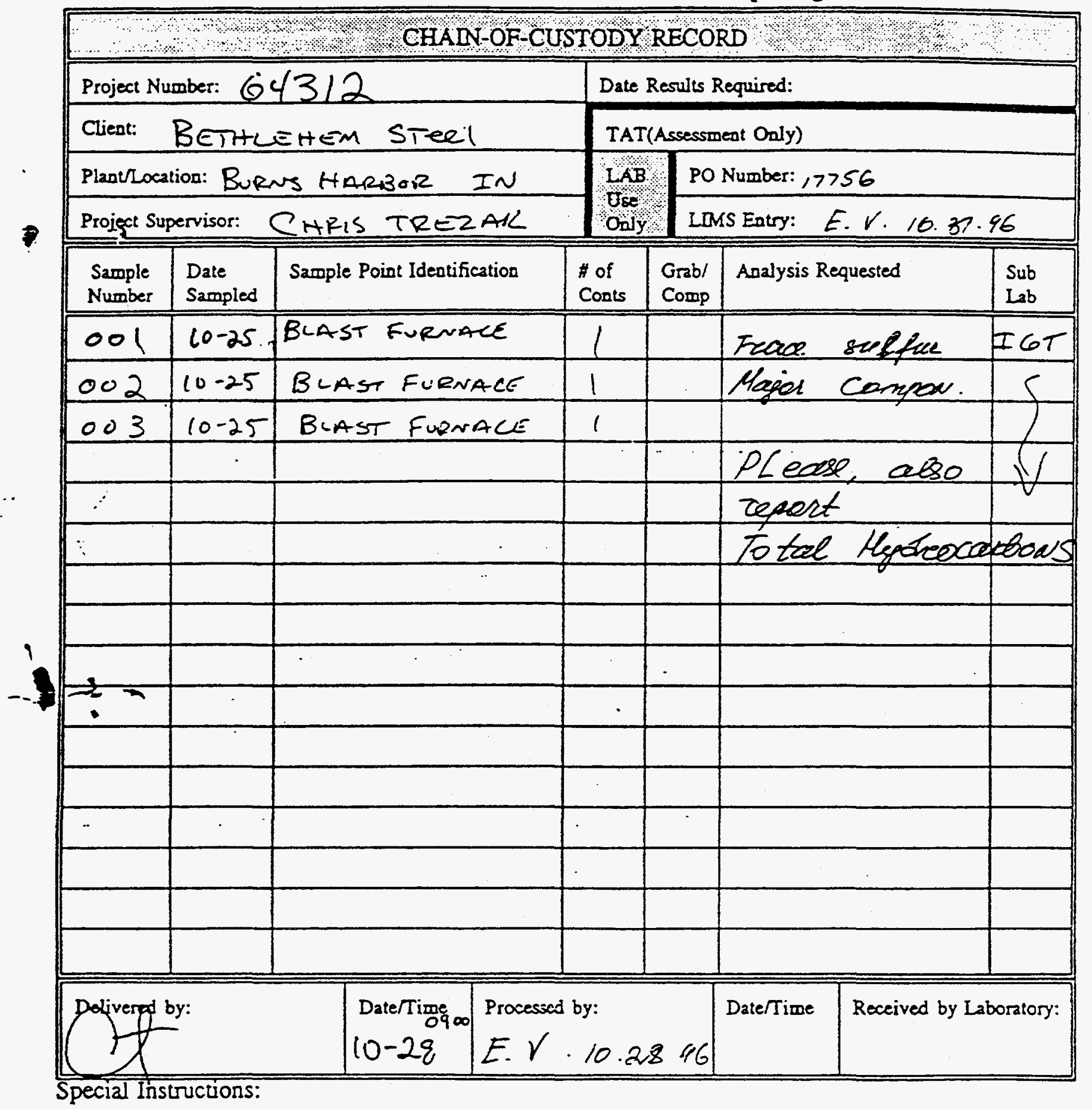




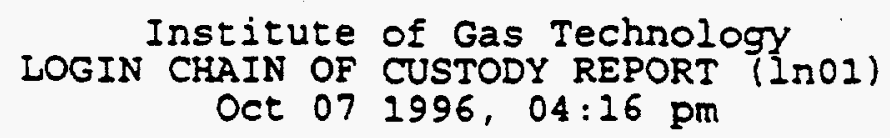

Login Number: L96-1496

Account: MOSTARDI PLATT MOstardi Platt Associates, Inc Project: MOST 96-1496

Saboratol $\%$ Claent $\%$ Collect Recelve

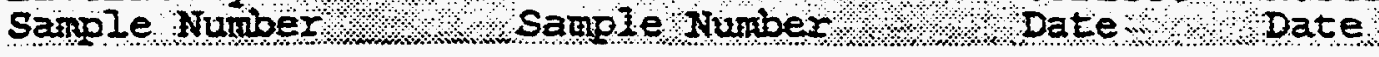

Due PR Date

\begin{tabular}{|c|c|c|c|c|c|}
\hline 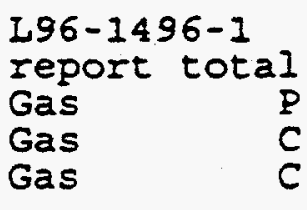 & $\begin{array}{l}\text { SAMPLE } \\
\text { hydrocarbons } \\
\text { LANDGAS2 } \\
\text { IANDGAS } \\
\text { SLFRTG }\end{array}$ & $64017-001$ & OCT-96 & $04-O C T-96$ & $\begin{array}{l}25-\text { OCT- } 96 \\
1 \text { Bottles }\end{array}$ \\
\hline $\begin{array}{l}\text { L96-1496-2 } \\
\text { report total } \\
\text { Gas } \\
\text { Gas }\end{array}$ & $\begin{array}{l}\text { SAMPLE } \\
\text { hydrocarbons } \\
\text { IANDGAS2 } \\
\text { IANDGAS } \\
\text { SLFRTG }\end{array}$ & $\begin{array}{r}64017-002 \\
\text { Hold }\end{array}$ & $\begin{array}{l}03-O C T-96 \\
-O C T-96\end{array}$ & $04-O C I-96$ & $\begin{array}{l}25 \text {-OCT- } 96 \\
1 \text { Botties }\end{array}$ \\
\hline $\begin{array}{l}\text { L96-1496-3 } \\
\text { report total } \\
\text { Gas }\end{array}$ & $\begin{array}{l}\text { SAMPLE } \\
\text { hydrocarbons } \\
\text { LANDGAS2 } \\
\text { IANDGAS } \\
\text { SLFRTG } \\
\text { ZZ SEH }\end{array}$ & $64017-003$ & $03-O C I-96$ & $04-O C T-96$ & $\begin{array}{l}25 \text {-OCT- } 96 \\
1 \text { Bozties }\end{array}$ \\
\hline
\end{tabular}

Page 1

Signature:

Date:

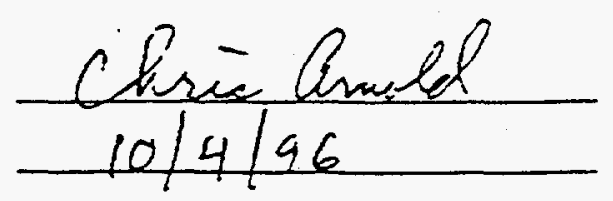




$$
\begin{aligned}
& \text { Institute of Gas Technology } \\
& \text { LOGIN CHAIN OF CUSTODY REPORT (InO1) } \\
& \text { OCt } 151996,10: 20 \mathrm{am}
\end{aligned}
$$

Login Number: L96-1512

Account: MOSTARDI PLATT Mostardi Platt Associates, Inc Project: MOST 96-1512

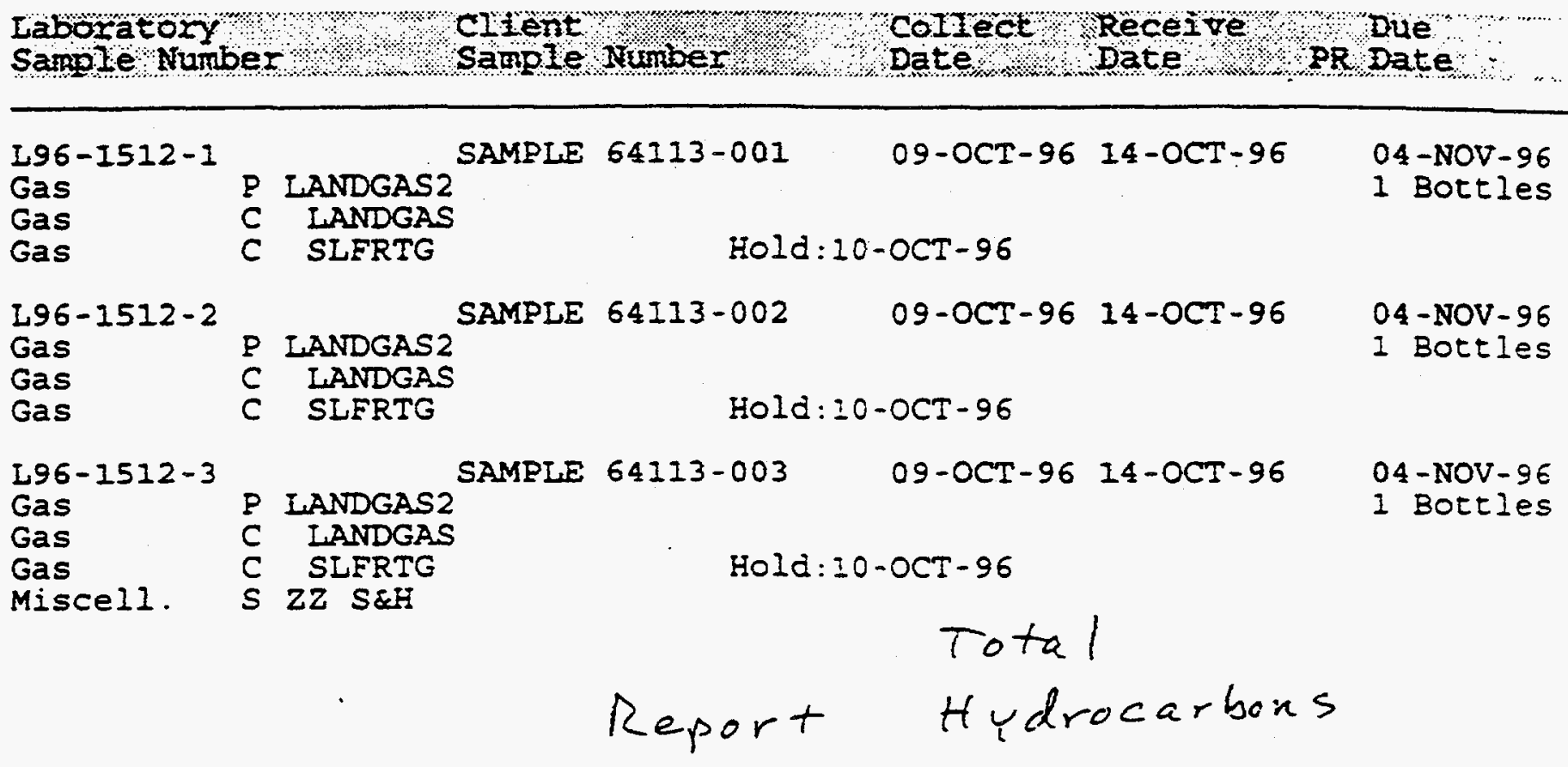

Page 1 signature: $\frac{\text { Chuilesed }}{\text { Date: }}$ 


\section{Institute of Gas Technology \\ LOGIN CHAIN OF CUSTODY REPORT (INOI) \\ OCt 22 2996, 04:34 pm}

Login Number: L96-1530

Account: MOSTARDI PLATT Mostardi Platt Associates, Inc Project: MOST 96-1530

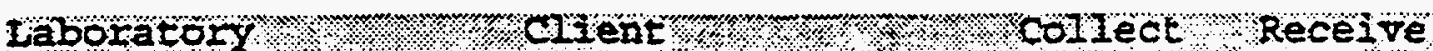

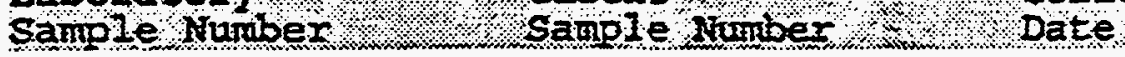
Date

Due

\begin{tabular}{|c|c|c|c|c|c|}
\hline $\begin{array}{l}\text { L96-1530-1 } \\
\text { report total } \\
\text { Gas } \\
\text { Gas }\end{array}$ & $\begin{array}{l}\text { SAMPLE } \\
\text { hYdrOCaIbOnS } \\
\text { IANDGAS2 } \\
\text { IANDGAS } \\
\text { SLFRTG }\end{array}$ & $\begin{array}{r}64202-001 \\
\text { HOIO }\end{array}$ & $17-0 C T-96$ & $21-$ OCT-96 & $\begin{array}{l}11 \text {-NOV- } 96 \\
1 \text { Bottles }\end{array}$ \\
\hline $\begin{array}{l}\text { L96-1530-2 } \\
\text { report } \\
\text { Gas }\end{array}$ & $\begin{array}{l}\text { SAMPLE } \\
\text { hydrocarbons } \\
\text { IANDGAS2 } \\
\text { IANDGAS } \\
\text { SLFRTG }\end{array}$ & $64202-002$ & $17-O C T-96$ & $21-O C T-96$ & $\begin{array}{l}11 \text {-NOV- } 96 \\
1 \text { Bottles }\end{array}$ \\
\hline $\begin{array}{l}\text { L96-1530-3 } \\
\text { report total } \\
\text { Gas } \\
\text { Gas } \\
\text { Gas } \\
\text { Miscell. }\end{array}$ & $\begin{array}{l}\text { SAMPLE } \\
\text { hYdrOCarbonS } \\
\text { IANDGAS2 } \\
\text { IANDGAS } \\
\text { SLFRTG } \\
\text { ZZ S\&H }\end{array}$ & $64202-003$ & $17-O C I-96$ & $21-O C T-96$ & $\begin{array}{l}11 \text {-NOV- } 96 \\
1 \text { Bottles }\end{array}$ \\
\hline
\end{tabular}

Page 1

Signature:

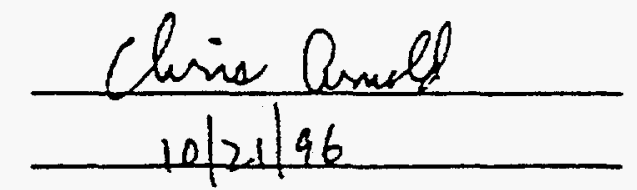




\section{Institute of Gas Technology \\ LOGIN CHAIN OF CUSTODY REPORT (InOI) \\ Oct 28 1996, 02:22 pm}

Login Number: L96-1538

Account: MOSTARDI PLATT Mostardi Plate Associates, Inc Project: MOST $96-1538$

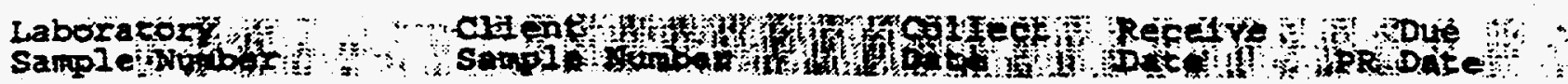

\begin{tabular}{|c|c|c|c|c|c|}
\hline $\begin{array}{lr}\text { L96-1538-1 } \\
\text { report total } \\
\text { Gas } & \text { P } \\
\text { Gas } & C \\
\text { Gas } & C\end{array}$ & $\begin{array}{l}\text { SAMPLE } \\
\text { hydrocarbonB } \\
\text { LANDGAS2 } \\
\text { LANDGAS } \\
\text { SLFRTG }\end{array}$ & $64312-002$ & $25-O C I-96$ & $2 B-O C T-96$ & $\begin{array}{l}18 \text {-NoV-96 } \\
1 \text { Bottles }\end{array}$ \\
\hline $\begin{array}{l}\text { L96-1538-2 } \\
\text { report total } \\
\text { Gas } \\
\text { Gas }\end{array}$ & $\begin{array}{l}\text { SAMPLE } \\
\text { hydrocarbonB } \\
\text { LANDGAS2 } \\
\text { IANDGAS } \\
\text { SLFRTG }\end{array}$ & $64312-002$ & $\begin{array}{l}25-O C T-96 \\
-O C T-96\end{array}$ & $28-O C T-96$ & $\begin{array}{l}18 \text {-NOV-96 } \\
1 \text { Bottles }\end{array}$ \\
\hline $\begin{array}{l}\text { L96-1538-3 } \\
\text { report total } \\
\text { Gas } \\
\text { Gas } \\
\text { Gas } \\
\text { Miscell. }\end{array}$ & $\begin{array}{l}\text { SAMPLE } \\
\text { hydrocarbons } \\
\text { IANDGAS2 } \\
\text { LANDGAS } \\
\text { SLERTG } \\
\text { 2Z SEH }\end{array}$ & $64312-003$ & OCT -96 & $28-O C T-96$ & $\begin{array}{l}18 \text {-NOV-96 } \\
1 \text { Bottles }\end{array}$ \\
\hline
\end{tabular}

\footnotetext{
Page 1

signature: Chai Anacel

Date:

$10 / 28 / 96$
} 


\section{Blast Furnace Granulated Coal Injection}

Environmental Monitoring Report

Appendix II - Wastewater Monitoring Summaries 


\section{Bethlehem Steel Corporation \\ Burns Harbor Division \\ Blast Furnace Closed Water Pump Station \\ Cold Well Monitoring Summary}

\begin{tabular}{|c|c|c|}
\hline $\begin{array}{l}02-\text { Oct-96 } \\
03-\text { Oct-96 } \\
04-\text { Oct-96 } \\
05-\text { Oct-96 } \\
06-\text { Oct-96 } \\
07-\text { Oct-96 } \\
08-\text { Oct-96 }\end{array}$ & 15.0 & 0.022 \\
\hline $\begin{array}{l}09-\text { Oct-96 } \\
10-\text { Oct-96 } \\
11-\text { Oct-96 } \\
12-\text { Oct-96 } \\
13-\text { Oct-96 } \\
14-\text { Oct-96 } \\
15-\text { Oct-96 }\end{array}$ & 15.4 & 0.012 \\
\hline $\begin{array}{l}16-\text { Oct-96 } \\
17-\text { Oct-96 } \\
18-\text { Oct-96 } \\
19-\text { Oct-96 } \\
20-\text { Oct-96 } \\
21-\text { Oct-96 } \\
22-\text { Oct-96 }\end{array}$ & 17.0 & $<0.005$ \\
\hline $\begin{array}{l}23-\text { Oct-96 } \\
24-\text { Oct-96 } \\
25-\text { Oct-96 } \\
26-\text { Oct-96 } \\
27-\text { Oct-96 } \\
28-\text { Oct-96 } \\
29-\text { Oct-96 } \\
30-\text { Oct-96 } \\
31-\text { Oct-96 }\end{array}$ & 47.9 & $<0.005$ \\
\hline $\begin{array}{l}\text { Average } \\
\text { Maximum } \\
\text { Minimum }\end{array}$ & $\begin{array}{l}23.8 \\
47.9 \\
15.0\end{array}$ & $\begin{array}{l}0.009 \\
0.022 \\
<0.005\end{array}$ \\
\hline Minimum & 15.0 & \\
\hline
\end{tabular}




\section{Bethlehem Steel Corporation Burns Harbor Division \\ Monitoring Station 011 Monitoring Summary}

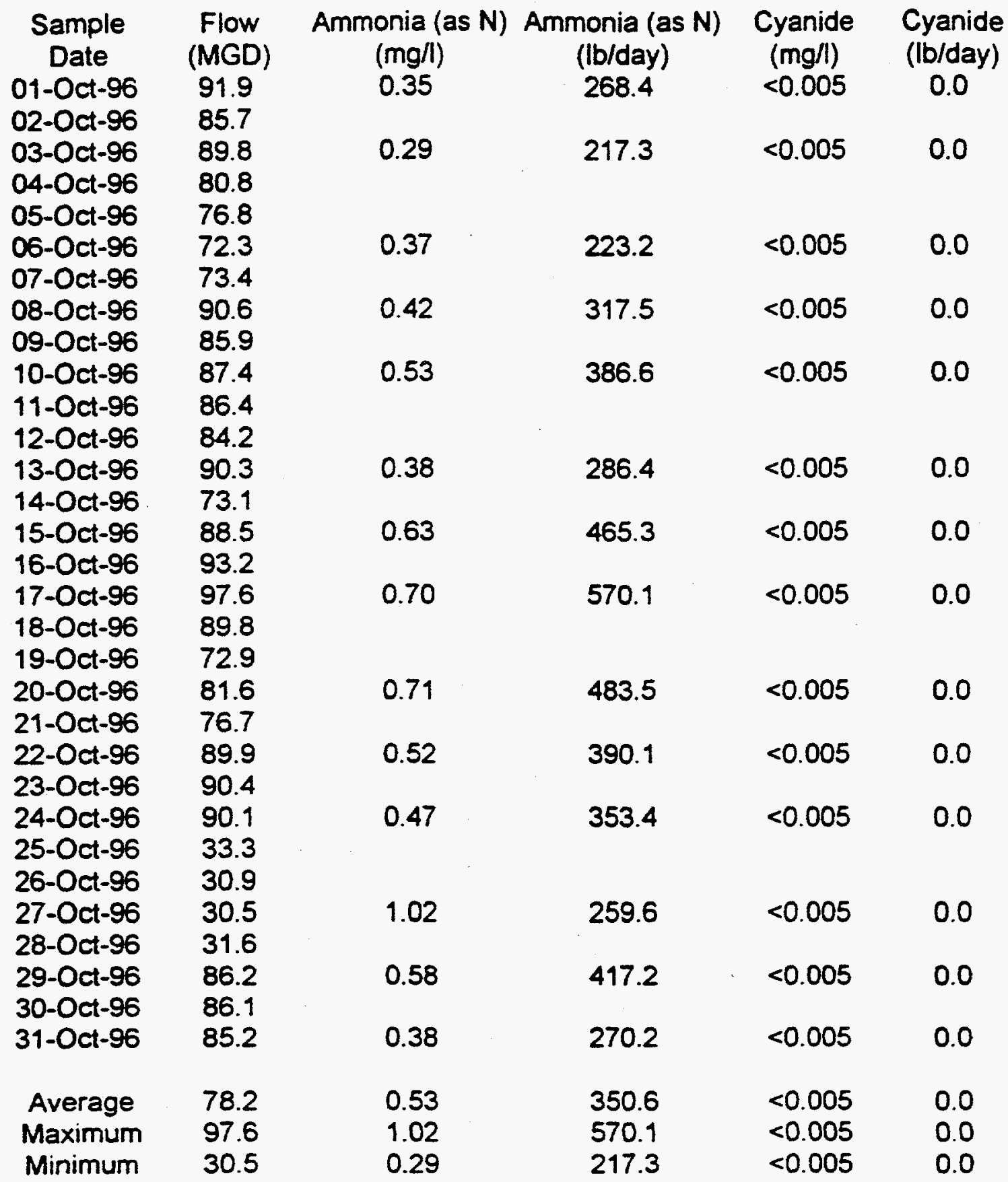


Bethlehem Steel Corporation

Burns Harbor Division

Outfall 001 Monitoring Summary

\begin{tabular}{|c|c|c|c|c|c|}
\hline $\begin{array}{c}\text { Sample } \\
\text { Date }\end{array}$ & $\begin{array}{l}\text { Flow } \\
\text { (MGD) }\end{array}$ & $\begin{array}{c}\text { Ammonia (as N) } \\
(\mathrm{mg} / 1)\end{array}$ & $\begin{array}{c}\text { Ammonia (as N) } \\
\text { (Ib/day) }\end{array}$ & $\begin{array}{c}\text { Cyanide } \\
(\mathrm{mg} / \mathrm{l})\end{array}$ & $\begin{array}{l}\text { Cyanide } \\
\text { (Ib/day) }\end{array}$ \\
\hline $\begin{array}{l}01-\text { Oct-96 } \\
02-\text { Oct-96 }\end{array}$ & $\begin{array}{l}138.8 \\
136.5\end{array}$ & & 335.9 & & \\
\hline 03-Oct-96 & 134.6 & 0.24 & 269.6 & & \\
\hline 04-Oct-96 & 130.1 & & & & \\
\hline 05-Oct-96 & 125.8 & & & & \\
\hline $\begin{array}{l}\text { 06-Oct-96 } \\
07-0 c t-96\end{array}$ & $\begin{array}{l}133.7 \\
125.7\end{array}$ & 0.30 & 334.7 & $<0.005$ & 0.0 \\
\hline 08-0ct-96 & 138.4 & 0.36 & 415.8 & & \\
\hline $09-0 c t-96$ & 135.8 & & & & \\
\hline 10-Oct-96 & 140.0 & 0.41 & 479.0 & & \\
\hline 11-Oct-96 & 132.0 & & & & \\
\hline 12-Oct-96 & 129.9 & & & & \\
\hline 13-Oct-96 & 132.8 & 0.37 & 410.0 & $<0.005$ & 0.0 \\
\hline $\begin{array}{l}\text { 14-0ct-96 } \\
15-0 c t-96\end{array}$ & $\begin{array}{l}128.3 \\
138.1\end{array}$ & 0.45 & 518.6 & & \\
\hline $16-0 c t-96$ & 144.4 & & & & \\
\hline 17-Oct-96 & 141.7 & 0.48 & 567.6 & . & \\
\hline $\begin{array}{l}18-0 c t-96 \\
19-0 c t-96\end{array}$ & $\begin{array}{l}138.7 \\
132.1\end{array}$ & & & & \\
\hline $\begin{array}{l}19-0 c t-96 \\
20-\text { Oct-96 }\end{array}$ & $\begin{array}{l}132.1 \\
125.0\end{array}$ & 0.40 & 417.3 & $<0,005$ & 0 \\
\hline $\begin{array}{l}20-0 \mathrm{ct}-96 \\
21-0 \mathrm{ct}-96\end{array}$ & 129.5 & & & & 0.0 \\
\hline 22-Oct-96 & 140.2 & 0.44 & 514.8 & & \\
\hline 23-Oct-96 & 135.2 & & & & \\
\hline $\begin{array}{l}24-\text { Oct-96 } \\
25-O c t-96\end{array}$ & $\begin{array}{c}134.0 \\
85.0\end{array}$ & 0.38 & 424.9 & & \\
\hline $\begin{array}{l}25-0 c t-96 \\
26-0 c t-96\end{array}$ & 82.9 & & & & \\
\hline 27-Oct-96 & 76.5 & 0.51 & 325.6 & $<0.005$ & 0.0 \\
\hline 28-Oct-96 & 102.8 & & & & \\
\hline 29-Oct-96 & 152.7 & 0.58 & 739.1 & & \\
\hline 30-Oct-96 & 125.3 & & & & \\
\hline 31-Oct-96 & 125.6 & 0.38 & 398.3 & & \\
\hline Average & 128.1 & 0.40 & 439.4 & $<0.005$ & 0.0 \\
\hline Maximum & 152.7 & 0.58 & 739.1 & $<0.005$ & 0.0 \\
\hline Minimum & 76.5 & 0.24 & 269.6 & $<0.005$ & 0.0 \\
\hline
\end{tabular}

